\title{
La gestione del paziente diabetico tra presente e futuro
}

\author{
Angelo Avogaro', Antonio C. Bossi ${ }^{2}$
}

${ }^{1}$ Dipartimento di Medicina, Università di Padova

${ }^{2}$ UOC Malattie Endocrine e Centro Regionale per il Diabete Mellito, AO Treviglio (Bg)

\section{Introduzione}

Il significativo ampliamento dell'armamentario terapeutico a disposizione dei clinici per il trattamento del diabete mellito comporta oggi la necessità di un approccio ragionato e multidisciplinare nella gestione del paziente con dismetabolismo glucidico.

La disponibilità di diverse classi farmacologiche pone le basi per un'ottimizzazione della strategia di trattamento del diabete in funzione delle caratteristiche cliniche individuali dei pazienti, rendendo tuttavia sempre più stringente la necessità di un aggiornamento professionale dinamico con lo scambio di esperienze cliniche e la condivisione delle conoscenze in tempo reale per un management efficace della malattia diabetica, al fine di prevenirne le complicanze croniche più frequenti e temibili quali le malattie cardiovascolari (CV) e renali.

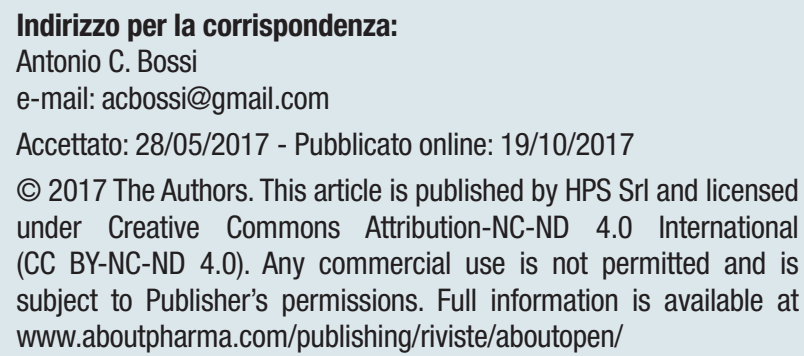

Le persone con diabete rappresentano il 25\% dei ricoverati in Unità di terapia intensiva coronarica, il $9 \%$ dei ricoverati per ictus ischemico e circa il $20 \%$ di quelli in terapia dialitica $[1,2]$.

Il diabete di tipo 2 si caratterizza per un importante carico aterosclerotico con progressione accelerata della placca ateromasica nonostante l'utilizzo di terapie mediche [3].

Diversi meccanismi sono stati chiamati in causa nella genesi del danno CV nei pazienti diabetici, quali iperglicemia e stress ossidativo, ipoglicemia, iperinsulinemia e resistenza insulinica.

È stato evidenziato come l'iperglicemia conduca attraverso una complessa via biochimica all'insorgenza di aterosclerosi. I meccanismi di tipo metabolico derivano da un'aumentata produzione di superossido a livello mitocondriale e sfociano nell'attivazione della via delle esosamine, la via dei polioli, quella della proteinchinasi $\mathrm{C}$ (PKC) e, in ultimo, nella generazione di advanced glycation endproducts (AGE). Produzione di superossido, PKC e AGE causano l'attivazione di vie di segnale mediate da MAPK (mitogen-activated protein kinase) e NF-kB (nuclear factor- $k B$ ), che a loro volta modulano l'espressione genica di citochine e fattori di crescita [4] (Figura 1).

Soprattutto nei soggetti obesi, largamente presenti nella popolazione diabetica, un ruolo importante è giocato dalle adipochine pro-infiammatorie prodotte dagli adi- 


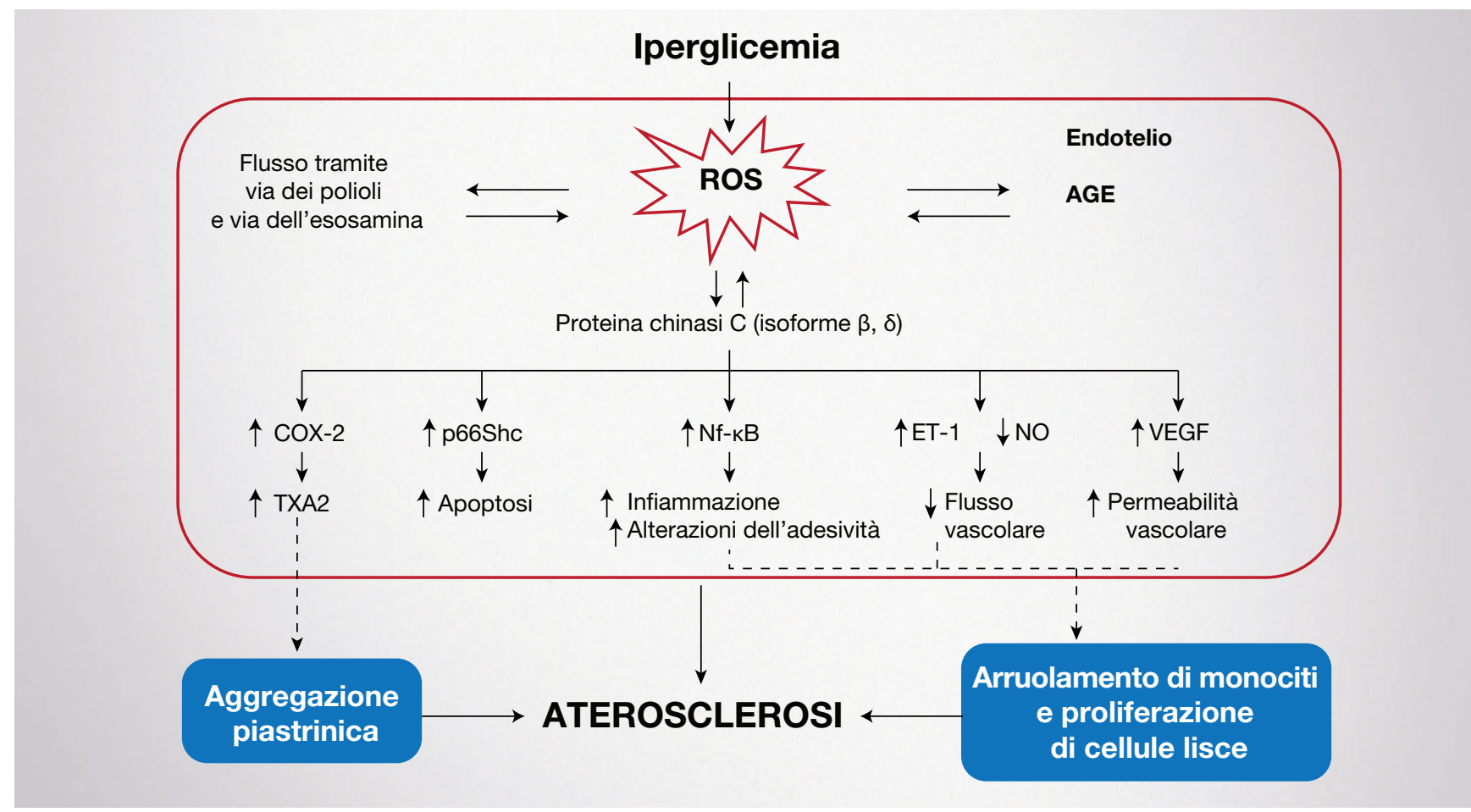

Figura 1. Meccanismi metabolici prodotti dall'iperglicemia (tratta dalla presentazione del prof. Avogaro). AGE, prodotti finali di glicazione avanzata; COX-2, ciclossigenasi-2; ET-1, endotelina-1; Nf-kB, fattore nucleare-kB; NO, ossido nitrico; ROS, specie reattive dell'ossigeno; TXA2, tromboxano A2; VEGF, fattore di crescita dell'endotelio vascolare

pociti. Tra le adipochine risulta particolarmente attiva la forma solubile della dipeptidil peptidasi 4 (sDPP4), che ha dimostrato di indurre infiammazione e proliferazione delle cellule muscolari lisce vascolari attraverso il recettore della proteasi attivata 2 (PAR-2) [5].

Un'altra noxa tissutale in grado di contribuire al danno CV è stata indicata nella cosiddetta NETosi, ossia una liberazione massiva di prodotti nucleari e granuli enzimatici [sostanze indicate complessivamente con il termine di neutrophil extracellular traps (NET)] osservabile nella morte cellulare dei neutrofili che è più marcata in condizioni patologiche come il diabete [6] $\mathrm{Si}$ è visto che alcuni biomarker sono correlati al rischio CV nel diabete di tipo 2. Nel dettaglio, è stato dimostrato che una riduzione di cellule progenitrici circolanti, dotate di proprietà vasculo-rigeneratrici, si associa a un aumento di circa due volte del rischio di eventi CV futuri e morte CV [7]. È stato in particolare mostrato come le cellule staminali CD34+ e CD133+ siano i fenotipi cellulari dotati del maggiore potere predittivo in tal senso.
Nei pazienti con diabete di tipo 2, per ogni aumento di un punto percentuale dell'emoglobina glicata (HbA1c) si osserva un incremento del rischio relativo di malattia CV del 18\% [8].

Per questo motivo, le ultime linee guida della European Society of Cardiology/European Association for the Study of Diabetes (ESC/EASD) enfatizzano l'importanza di un controllo glicemico stretto nei pazienti con dismetabolismo glucidico [9].

Un valore di $\mathrm{HbA} 1 \mathrm{c}<7 \%$ viene indicato come obiettivo da raggiungere per massimizzare i benefici CV della terapia ipoglicemizzante [10].

Tuttavia, per quanto sia indiscusso che il rischio CV aumenti parallelamente ai valori di HbA1c, i trial di intervento volti a determinare se un controllo glicemico intensivo si associ a una riduzione del rischio di malattia CV hanno prodotto risultati controversi [11]. Esiste quello che viene definito come il "paradosso macrovascolare", ovvero l'insufficiente riduzione delle complicanze macroangiopatiche di un livello pa- 


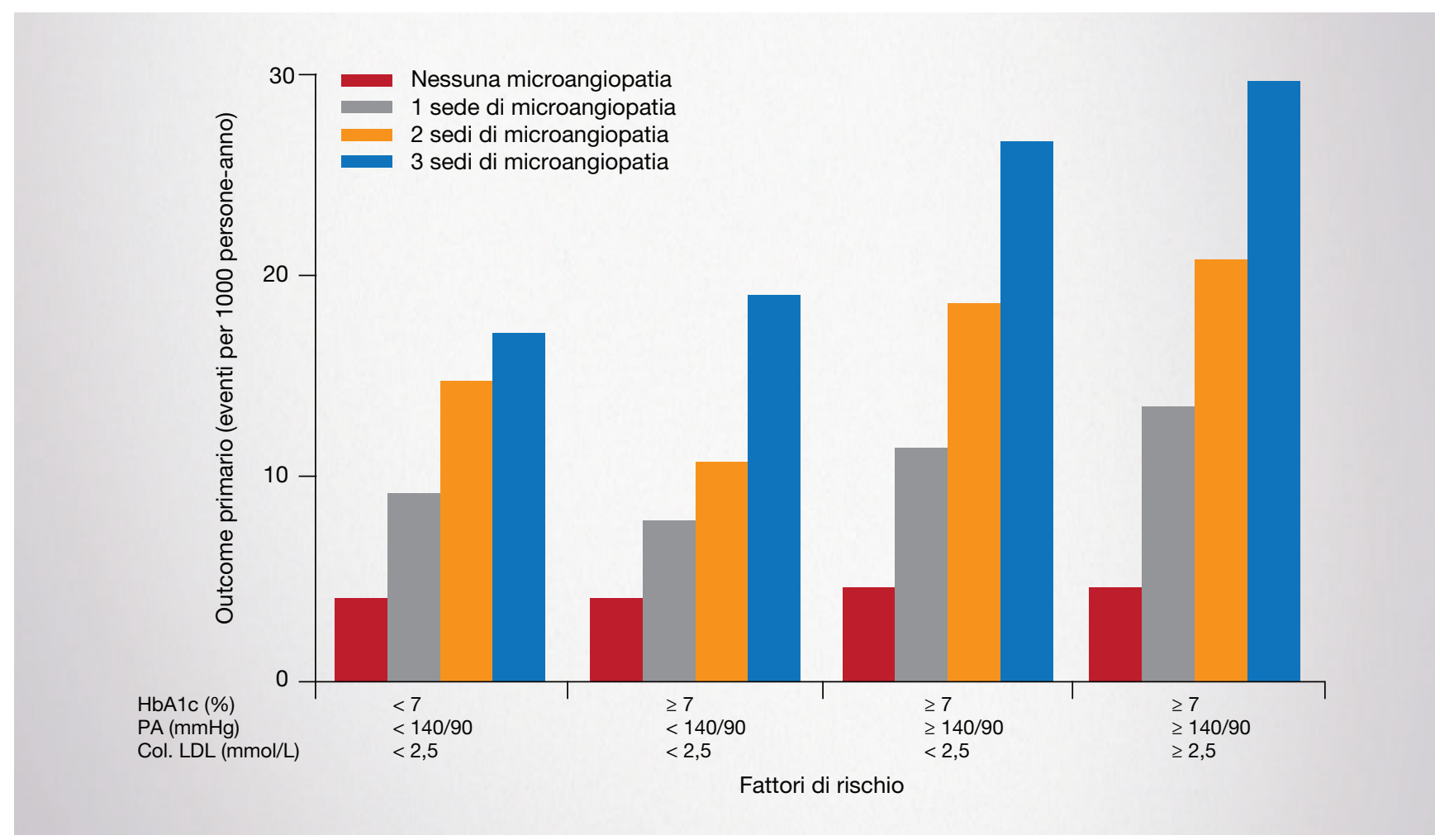

Figura 2. Correlazione tra malattia microvascolare ed eventi cardiovascolari maggiori (modificata da [13]). HbA1C, emoglobina glicata; PA, pressione arteriosa

ragonabile a quello osservato per le complicanze microangiopatiche nonostante uguali riduzioni paragonabili dei valori di HbA1c. Una possibile spiegazione di questo fenomeno può essere ricercata nella natura multifattoriale del rischio CV nel diabete, come evidenziato dai risultati dello studio STENO-2 [12].

Corollario di questa osservazione è il fatto che agenti farmacologici dotati di effetti che si estendono oltre a quello ipoglicemizzante possono avere un impatto differente sul rischio CV.

È comunque ormai chiaro come micro- e macroangiopatia rappresentino un continuum della malattia vascolare diabetica. La microangiopatia è, infatti, un fattore di rischio cruciale per la progressione della macroangiopatia [13]. È stata dimostrata una forte correlazione tra presenza di malattia microvascolare ed eventi CV maggiori (major adverse cardiovascular events, MACE) nei pazienti con diabete di tipo 2. In particolare, si è evidenziata un'associazione tra eventi CV e retinopatia [hazard ratio (HR) 1,39; intervallo di confidenza (IC) 95\% 1,09-1,76], neuropatia periferica (HR 1,40; IC 95\% 1,19-1,66) e nefropatia (HR 1,35; IC 95\% 1,15-1,58). Nei soggetti con una, due o tre patologie microvascolari rispetto a nessuna, gli hazard ratio aggiustati per il primo evento $\mathrm{CV}$ si sono dimostrati rispettivamente pari a 1,32 (IC 95\% 1,16-1,50), 1,62 (IC 95\% 1,42-1,85) e 1,99 (IC 95\% 1,70-2,34) (Figuira 2).

Nella scelta del trattamento farmacologico del diabete occorre pertanto considerare le capacità della terapia di incidere sulla storia naturale della malattia con particolare riferimento agli aspetti di protezione cardiovascolare e renale.

\section{Nuove opportunità di protezione cardiovascolare per \\ il paziente con diabete di tipo 2}

La riduzione del rischio $\mathrm{CV}$ nel paziente diabetico rappresenta un obiettivo primario, al pari del control- 
lo glicemico. La presenza in particolare di diabete e di una $\mathrm{O}$ più comorbilità $\mathrm{CV}$ esercita un effetto moltiplicatore sulla probabilità di decesso. Sono al proposito illuminanti i dati dell'Emerging Risk Factors Collaboration, relativi a 689.300 soggetti di 91 coorti analizzati in un periodo compreso tra il 1960 e il 2007 [14] In questo lasso di tempo, su un totale di circa 9 milioni di pazienti/anno di osservazione, si sono verificati 128.843 morti.

Nei pazienti che non hanno né diabete, né ictus, né infarto, la mortalità è di 6,8 per 1000 persone-anno; la presenza di diabete o di una delle patologie CV raddoppia l'hazard ratio di decesso per tutte le cause; la presenza contemporanea di diabete e una comorbilità CV quadruplica il rischio di morte per tutte le cause e la co-presenza di diabete, ictus e infarto aumenta di quasi sette volte il tasso di mortalità per tutte le cause (Figura 3) [14].

I dati dello studio sono stati confrontati con quelli del UK Biobank, in cui 499.808 partecipanti sono stati seguiti nel periodo 2006-2010, riscontrando complessivamente 7.995 morti all'ultimo follow-up. La stratificazione dei pazienti in base alla presenza di diabete e/o comorbilità CV ha permesso di evidenziare tassi di mortalità con una dinamica del tutto simile a quella riscontrata dall'Emerging Risk Factors Collaboration, corroborando il riscontro sia di un rischio di mortalità aumentato dalla semplice presenza di diabete sia di un effetto moltiplicatore legato alla presenza simultanea di una o più malattie CV. Questi dati sono in linea con quelli evidenziati in altri ampi studi di popolazione come il Physicians' Health Study e il Nurses Health Study.

Gli autori dell'indagine Emerging Risk Factors Collaboration hanno stimato che a 60 anni di età gli uomini con due delle condizioni cardiometaboliche considerate hanno in media un'aspettativa di vita ridotta di 12 anni, mentre quelli con tutte e tre le condizioni patologiche vedono ridursi la propria aspettativa di vita di 14 anni. Nelle donne la situazione è leggermente peggiore: l'aspettativa di vita a 60 anni si riduce rispettivamente di 13 e di 16 anni in presenza di due o di tre patologie cardiovascolari. Il calcolo relativo a pazienti di età più giovane fa dipingere un quadro a tinte ancora più fosche. A 40 anni di età la presenza simultanea di tre condizioni patologiche $\mathrm{CV}$ fa perdere ben 23 anni di vita attesa, che diventano 20 a 50 anni di età. Anche dopo i 70 anni l'aspettativa di vita viene ridotta in misura importante dalla presenza di diabete da solo o in associazione alle altre comorbilità CV (Tabella 1) [14]. I trattamenti a disposizione per la cura del diabete devono dunque essere in grado tanto di correggere la glicemia quanto di migliorare gli endpoint $\mathrm{CV}$ nei pazienti diabetici.

L'algoritmo decisionale per la terapia nel diabete di tipo 2 secondo gli standard italiani redatti dall'Associazione Medici Diabetologi/Società Italiana di Diabetologia (AMD/SID) è riportato nella Figura 4 [15]. Sono stati condotti diversi trial che hanno misurato outcome CV dei farmaci antidiabetici. La metformina

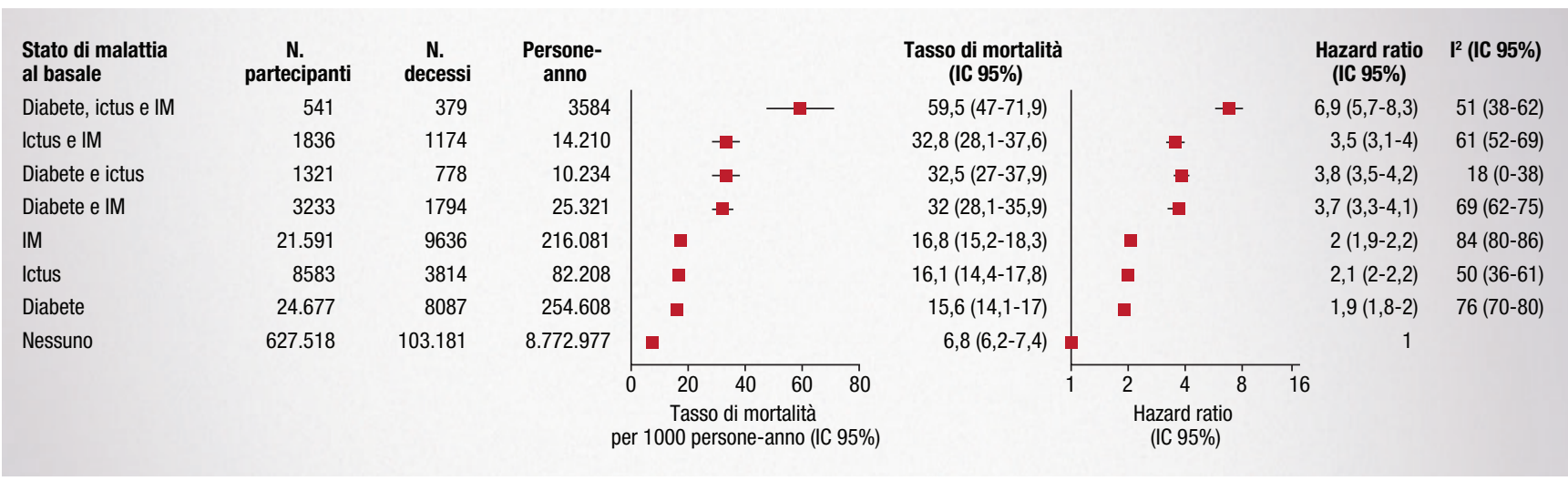

Figura 3. Tasso di mortalità per tutte le cause in base alla presenza di diabete e comorbilità cardiovascolari (modificata da [14]). IM, infarto miocardico 
Tabella 1. Anni di vita persi in funzione della presenza di diabete e/o patologie cardiovascolari (modificata da [14]).

\begin{tabular}{|c|c|c|c|c|c|c|c|c|c|c|}
\hline \multirow{2}{*}{ Stato di malattia al basale } & \multicolumn{10}{|c|}{ Età (anni) } \\
\hline & 40 & 45 & 50 & 55 & 60 & 65 & 70 & 75 & 80 & 85 \\
\hline \multicolumn{11}{|l|}{ Uomini } \\
\hline $\begin{array}{l}\text { Diabete, ictus e infarto } \\
\text { miocardico }\end{array}$ & 22,9 & 21,7 & 19,8 & 17,3 & 14,4 & 11,2 & 8,1 & 5,2 & 2,9 & 1,2 \\
\hline Ictus e infarto miocardico & 21,5 & 20,1 & 18,1 & 15,6 & 12,9 & 10,1 & 7,4 & 4,8 & 2,7 & 1,2 \\
\hline Diabete e ictus & 16,3 & 15,6 & 14,5 & 13,1 & 11,2 & 9,1 & 6,8 & 4,6 & 2,6 & 1,2 \\
\hline $\begin{array}{l}\text { Diabete e infarto } \\
\text { miocardico }\end{array}$ & 16,8 & 16 & 14,8 & 13,1 & 11,2 & 9 & 6,7 & 4,5 & 2,6 & 1,2 \\
\hline Solo infarto miocardico & 10 & 9,6 & 8,9 & 8 & 7 & 5,7 & 4,4 & 3,1 & 1,9 & 0,9 \\
\hline Solo ictus & 8,8 & 8,6 & 8,1 & 7,5 & 6,6 & 5,6 & 4,4 & 3,1 & 1,9 & 0,9 \\
\hline Solo diabete & 7,9 & 7,6 & 7,2 & 6,5 & 5,7 & 4,8 & 3,7 & 2,7 & 1,6 & 0,8 \\
\hline \multicolumn{11}{|l|}{ Donne } \\
\hline $\begin{array}{l}\text { Diabete, ictus e infarto } \\
\text { miocardico }\end{array}$ & 20,1 & 19,8 & 19,1 & 17,7 & 15,7 & 13 & 9,9 & 6,7 & 3,9 & 1,8 \\
\hline Ictus e infarto miocardico & 13 & 12,8 & 12,3 & 11,5 & 10,4 & 9 & 7,2 & 5,2 & 3,3 & 1,6 \\
\hline Diabete e ictus & 16,5 & 16,2 & 15,5 & 14,5 & 13 & 11,1 & 8,7 & 6,2 & 3,8 & 1,9 \\
\hline $\begin{array}{l}\text { Diabete e infarto } \\
\text { miocardico }\end{array}$ & 19,9 & 19,2 & 18,1 & 16,4 & 14,3 & 11,8 & 9,1 & 6,4 & 3,8 & 1,8 \\
\hline Solo infarto miocardico & 6,5 & 6,4 & 6,2 & 5,9 & 5,5 & 4,9 & 4,1 & 3,1 & 2 & 1 \\
\hline Solo ictus & 7,4 & 7,4 & 7,2 & 7 & 6,5 & 5,9 & 5 & 3,8 & 2,5 & 1,3 \\
\hline Solo diabete & 8,2 & 8,1 & 7,8 & 7,3 & 6,7 & 5,9 & 4,8 & 3,6 & 2,4 & 1,2 \\
\hline
\end{tabular}

è considerato il farmaco di prima scelta sulla base dei risultati della sotto-analisi dello studio UKPDS che ha mostrato una riduzione del 39\% di infarto miocardico anche se in un limitato numero di pazienti diabetici sovrappeso. Tuttavia, recentemente questo farmaco è stato messo in discussione sia in merito all'efficacia che agli outcome [16].

Nello studio ADVANCE (Action in Diabetes and Vascular Disease: Preterax and Diamicron Modified Release Controlled Evaluation), il controllo glicometabolico stretto con gliclazide a rilascio modificato ha ridotto l'incidenza di eventi combinati micro- e macroangiopatici in virtù di una riduzione dell'incidenza di una nuova nefropatia o del peggioramento di una nefropatia pre-esistente [17]. Una metanalisi dello studio STOP-NIDDM ha concluso che la riduzione di rischio CV con acarbosio, osservata in soggetti con alterata tolleranza al glucosio, risulta controversa in pazienti con diabete di tipo $2[18,19]$.

Nel Prospective Pioglitazone Clinical Trial in Ma- crovascular Events (PROactive) un tiazolidinedione, il pioglitazone, ha ridotto del 16\% l'outcome secondario pre-specificato di infarto miocardico, ictus e morte $\mathrm{CV}$ ma ha aumentato i casi di scompenso cardiaco [20].

Indicazioni utili sugli outcome $\mathrm{CV}$ arrivano da sette trial oggi a disposizione, di cui tre sui DPP4 inibitori [21-23], tre su agonisti recettoriali del glucagon-like peptide 1 (GLP1-RA) [24-26] e uno su un inibitore del cotrasportatore sodio-glucosio 2 (SGLT2) [27] (Figura 5).

L'intervento farmacologico con DPP4 inibitori è risultato nei trial di sicurezza cardiovascolare (Cardiovascular Outcome Trials, CVOT) sostanzialmente neutro in termini di prevenzione CV. I risultati degli studi clinici condotti con saxagliptin, alogliptin (nella popolazione di pazienti dopo sindrome coronarica acuta recente) e sitagliptin hanno dimostrato curve di sopravvivenza sovrapponibili per i pazienti in trattamento con farmaco attivo e per quelli trattati con placebo. Lo studio SAVOR TIMI condotto con saxaglip- 


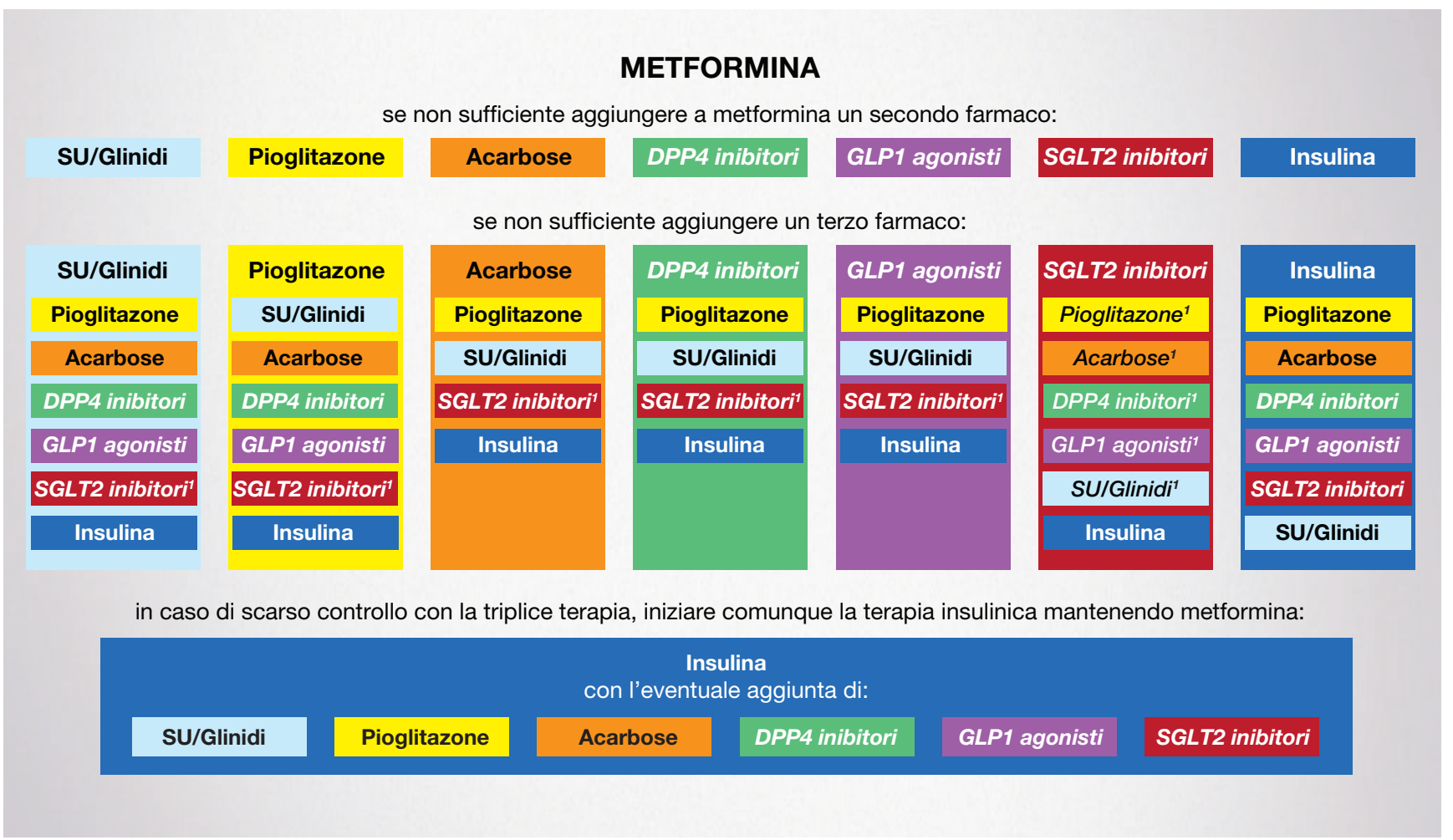

Figura 4. Schema generale per la terapia farmacologica del diabete. In grassetto le combinazioni rimborsate, in grassetto corsivo le combinazioni rimborsate solo con piano terapeutico specialistico, in corsivo le combinazioni indicate ma non rimborsate. "Indicazioni approvate da EMA, per le quali AIFA ha deciso di non concedere la rimborsabilità (modificata da [15]). DPP4, enzima dipeptidil-peptidasi-4; GLP1, glucagon-like peptide 1; SGLT2, cotrasportatore di sodio-glucosio 2; SU, sulfoniluree

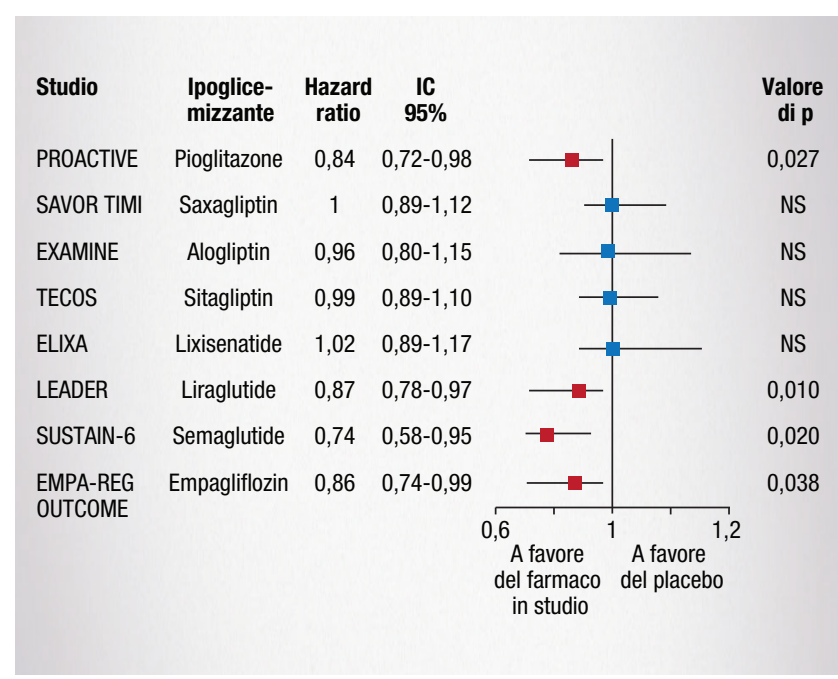

Figura 5. Effetto di DPP4 inibitori, GLP1-RA e SGLT2 inibitore su eventi cardiovascolari maggiori (MACE) a 3 punti (mortalità cardiovascolare, infarto miocardico non fatale e ictus non fatale) nel diabete di tipo 2 (elaborata da [20-27]). NS, non significativo tin ha riscontrato un eccesso di ospedalizzazioni per scompenso cardiaco, ma non un aumento di mortalità per cardiovasculopatia. Una successiva metanalisi ha concluso che i DPP4 inibitori hanno un effetto neutro sullo scompenso cardiaco [28].

Liraglutide e semaglutide hanno dimostrato effetti positivi in termini di protezione $\mathrm{CV}$, mentre lixisenatide ha dato risultati neutri in tal senso. Empagliflozin ha ottenuto risultati estremamente positivi in termini di riduzione di eventi $\mathrm{CV}$.

È da sottolineare che i risultati dei trial più recenti, l'EMPA-REG OUTCOME con empagliflozin e il LEADER con liraglutide, hanno suscitato un notevole entusiasmo nella comunità diabetologica e cardiologica. EMPA-REG OUTCOME ha valutato la sicurezza del SGLT2 inibitore empagliflozin in pazienti con diabete mellito di tipo 2 e indagato i potenziali effetti cardioprotettivi del farmaco con un focus particolare sugli 
outcome microvascolari. Lo studio, randomizzato, in doppio cieco e controllato contro placebo, ha incluso 7.034 diabetici con malattie CV note (infarto miocardico, coronaropatia, angina instabile, ictus, scompenso cardiaco, arteriopatia obliterante periferica) e con un controllo glicemico non ottimale (HbA1c tra $7 \%$ e 10\%). Il trial ha avuto l'obiettivo di esaminare gli effetti a lungo termine di empagliflozin in aggiunta alla terapia standard su morbilità e mortalità CV in pazienti diabetici ad alto rischio di eventi cardiocircolatori. Si tratta quindi di uno studio di prevenzione secondaria che ha escluso i pazienti con insufficienza renale severa [filtrato glomerulare stimato (eGFR) $<30 \mathrm{ml} / \mathrm{min} / 1,73 \mathrm{~m}^{2}$ ]. È opportuno ricordare che il farmaco non può essere prescritto con valori di eGFR $<60 \mathrm{ml} / \mathrm{min}$, tuttavia può essere continuato fino a valori di $45 \mathrm{ml} / \mathrm{min}$ qualora il paziente abbia iniziato la terapia con valori di eGFR $\geq 60 \mathrm{ml} / \mathrm{min}$ [29].

Nel trial sono stati somministrati due dosaggi del farmaco, $10 \mathrm{mg}$ e $25 \mathrm{mg}$. Pur non trattandosi di uno studio treat to target, lo sperimentatore era comunque stimolato a ottenere il migliore controllo glicemico possibile in pazienti già in terapia ipoglicemizzante. I pazienti inclusi nello studio erano trattati al basale con un nutrito numero di farmaci $\mathrm{CV}$, a causa dell'elevato profilo di rischio CV (Tabelle 2 e 3 ).

I farmaci per la terapia antidiabetica di base più utilizzati sono stati: metformina (circa il 74\%), sulfoniluree

Tabella 2. Terapie antipertensive assunte al basale dai pazienti nel trial EMPA-REG OUTCOME (modificata da [27]). ACE, enzima di conversione dell'angiotensina; ARB, antagonista recettoriale dell'angiotensina II

\begin{tabular}{|l|c|c|c|}
\hline & $\begin{array}{c}\text { Placebo } \\
(\mathbf{n}=\mathbf{2 3 3 3})\end{array}$ & $\begin{array}{c}\text { Empagliflozin } \\
\mathbf{1 0} \mathbf{m g}(\mathbf{n}=\mathbf{2 3 4 5})\end{array}$ & $\begin{array}{c}\text { Empagliflozin } \\
\mathbf{2 5} \mathbf{~ m g ~ ( n = ~ 2 3 4 2 )}\end{array}$ \\
\hline Terapia antipertensiva & $2221(95,2 \%)$ & $2227(95 \%)$ & $2219(94,7 \%)$ \\
\hline ACE inibitori/ARB & $1868(80,1 \%)$ & $1896(80,9 \%)$ & $1902(81,2 \%)$ \\
\hline Beta-bloccanti & $1498(64,2 \%)$ & $1530(65,2 \%)$ & $1526(65,2 \%)$ \\
\hline Diuretici & $988(42,3 \%)$ & $1036(44,2 \%)$ & $1011(43,2 \%)$ \\
\hline Calcioantagonisti & $788(33,8 \%)$ & $781(33,3 \%)$ & $748(31,9 \%)$ \\
\hline Antagonisti recettoriali dei mineralcorticoidi & $136(5,8 \%)$ & $157(6,7 \%)$ & $148(6,3 \%)$ \\
\hline Inibitori della renina & $19(0,8 \%)$ & $16(0,7 \%)$ & $11(0,5 \%)$ \\
\hline Altro & $191(8,2 \%)$ & $193(8,2 \%)$ & $190(8,1 \%)$ \\
\hline
\end{tabular}

Tabella 3. Farmaci cardiovascolari assunti al basale dai pazienti nel trial EMPA-REG OUTCOME (modificata da [27]).

\begin{tabular}{|l|c|c|c|}
\hline & $\begin{array}{c}\text { Placebo } \\
(\mathbf{n}=\mathbf{2 3 3 3})\end{array}$ & $\begin{array}{c}\text { Empagliflozin } \\
\mathbf{1 0} \mathbf{~ m g ~ ( n = ~ 2 3 4 5 ) ~}\end{array}$ & $\begin{array}{c}\text { Empagliflozin } \\
\mathbf{2 5} \mathbf{~ m g ~ ( n ~ = ~ 2 3 4 2 ) ~}\end{array}$ \\
\hline Ipolipemizzanti & $1864(79,9 \%)$ & $1926(82,1 \%)$ & $1894(80,9 \%)$ \\
\hline Statine & $1773(76 \%)$ & $1827(77,9 \%)$ & $1803(77 \%)$ \\
\hline Fibrati & $199(8,5 \%)$ & $214(9,1 \%)$ & $217(9,3 \%)$ \\
\hline Ezetimibe & $81(3,5 \%)$ & $95(4,1 \%)$ & $94(4 \%)$ \\
\hline Niacina & $35(1,5 \%)$ & $56(2,4 \%)$ & $35(1,5 \%)$ \\
\hline Altro & $175(7,5 \%)$ & $172(7,3 \%)$ & $193(8,2 \%)$ \\
\hline Anticoagulanti e antiaggreganti & $2090(89,6 \%)$ & $2098(89,5 \%)$ & $2064(88,1 \%)$ \\
\hline Acido acetilsalicilico & $1927(82,6 \%)$ & $1939(82,7 \%)$ & $1937(82,7 \%)$ \\
\hline Clopidogrel & $249(10,7 \%)$ & $253(10,8 \%)$ & $241(10,3 \%)$ \\
\hline Antagonisti della vitamina K & $156(6,7 \%)$ & $141(6 \%)$ & $125(5,3 \%)$ \\
\hline
\end{tabular}


(oltre il 42\%), insulina (quasi il 50\% della popolazione). Metà dei partecipanti aveva una storia di infarto e il $10 \%$ presentava scompenso cardiaco. Da notare che in questa popolazione solo il $10 \%$ presentava una coronaropatia monovasale, una ulteriore conferma dell'esteso coinvolgimento dell'albero coronarico nel paziente diabetico.

Più del 95\% dei soggetti inclusi nello studio era in terapia antipertensiva (oltre l' $80 \%$ con un ACE inibitore/sartano, il 65\% con un beta-bloccante, oltre il 40\% con diuretici e 1 su 3 con un calcioantagonista). Tre su quattro erano in terapia con statine e oltre $1{ }^{\prime} 82 \%$ con acido acetilsalicilico.

È da rimarcare che in questa popolazione già trattata in modo aggressivo per ridurre il rischio CV, l'aggiunta di empagliflozin ha migliorato leggermente il controllo glicometabolico, riducendo l'HbA1c dello 0,3-0,4\% rispetto alla terapia standard modulata in modo dinamico sulle esigenze del singolo paziente, e ha abbattuto in tre anni del 38\% la mortalità CV, del $32 \%$ la mortalità totale e del 35\% le ospedalizzazioni per scompenso cardiaco.

Un aspetto del trial è particolarmente significativo e riguarda l'effetto estremamente rapido di empagliflo- zin sull'endpoint composito di mortalità CV, infarto miocardico e ictus non fatali, con curve che si separano dopo poche settimane e si mantengono divergenti fino alla fine dello studio (Figura 6).

Scorporando la mortalità CV per i due dosaggi del farmaco, si riconferma la precocità dell'effetto (Figura 7). Anche in merito alle ospedalizzazioni per scompenso cardiaco, le curve relative al trattamento con placebo ed empagliflozin si divaricano dopo poche settimane di trattamento e divergono ulteriormente nel tempo senza una differenza significativa tra i due dosaggi, sia per i soggetti con nuova insorgenza di insufficienza cardiaca sia in quelli con eventi pregressi (Figura 8). Recentemente è stato pubblicato anche lo studio CANVAS con canagliflozin che è consistito di 2 studi: il CANVAS $(n=4330)$ disegnato per valutare la sicurezza cardiovascolare di canagliflozin e il CANVAS-Renal (CANVAS-R) $(\mathrm{n}=5812)$ disegnato non solo per stabilire la sicurezza CV ma anche gli effetti sulla microalbuminuria. I due studi sono stati analizzati congiuntamente. In pazienti seguiti per 5,6 anni il trattamento con canagliflozin comportava una significativa riduzione dell'endpoint primario (26,9 vs 31,5 pazienti con evento per 1000 persone-anno (HR 0,86; IC 95\%

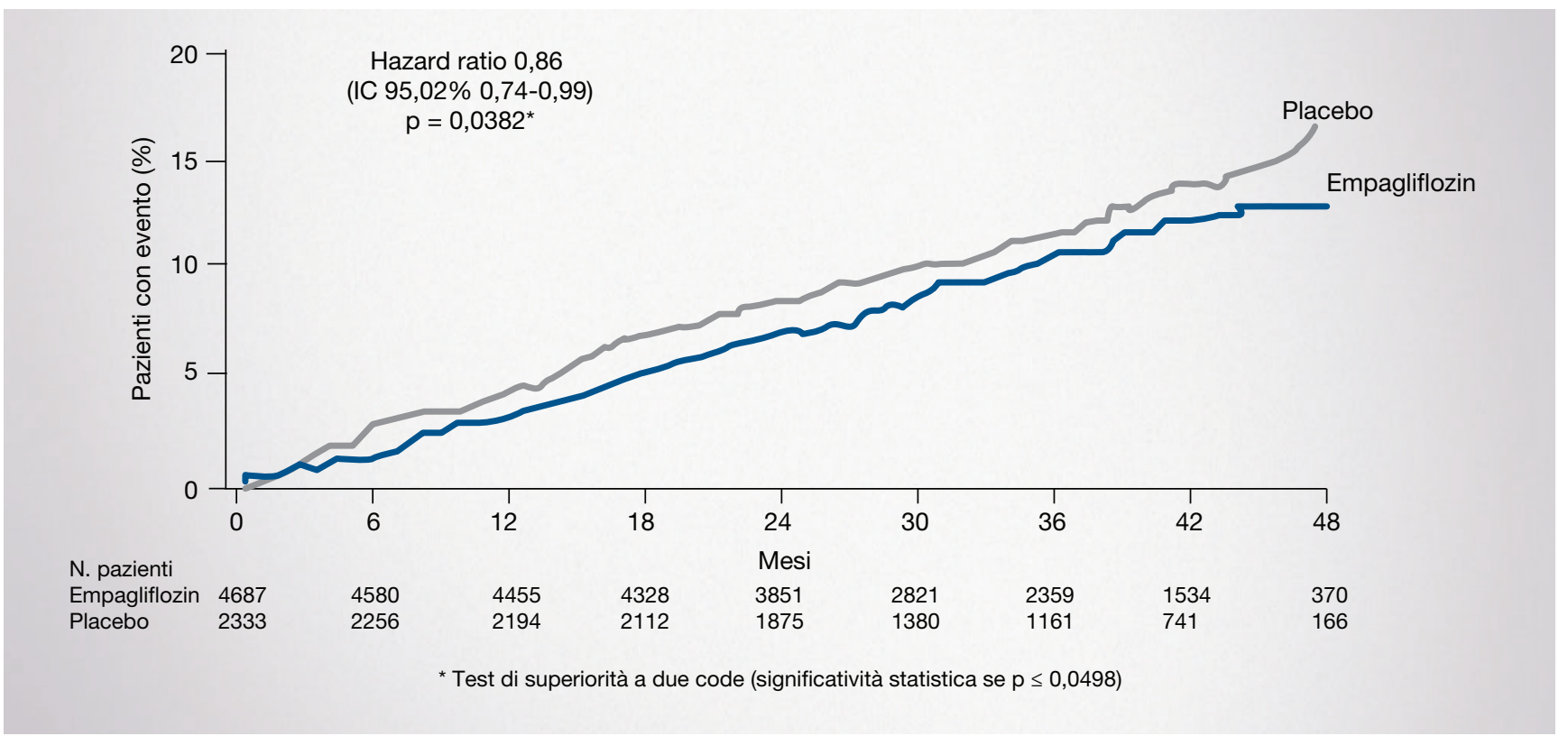

Figura 6. Outcome primario dello studio EMPA-REG OUTCOME: 3 punti MACE (mortalità cardiovascolare, infarto miocardico non fatale, ictus non fatale) (modificata da [27]). MACE, eventi cardiovascolari maggiori 


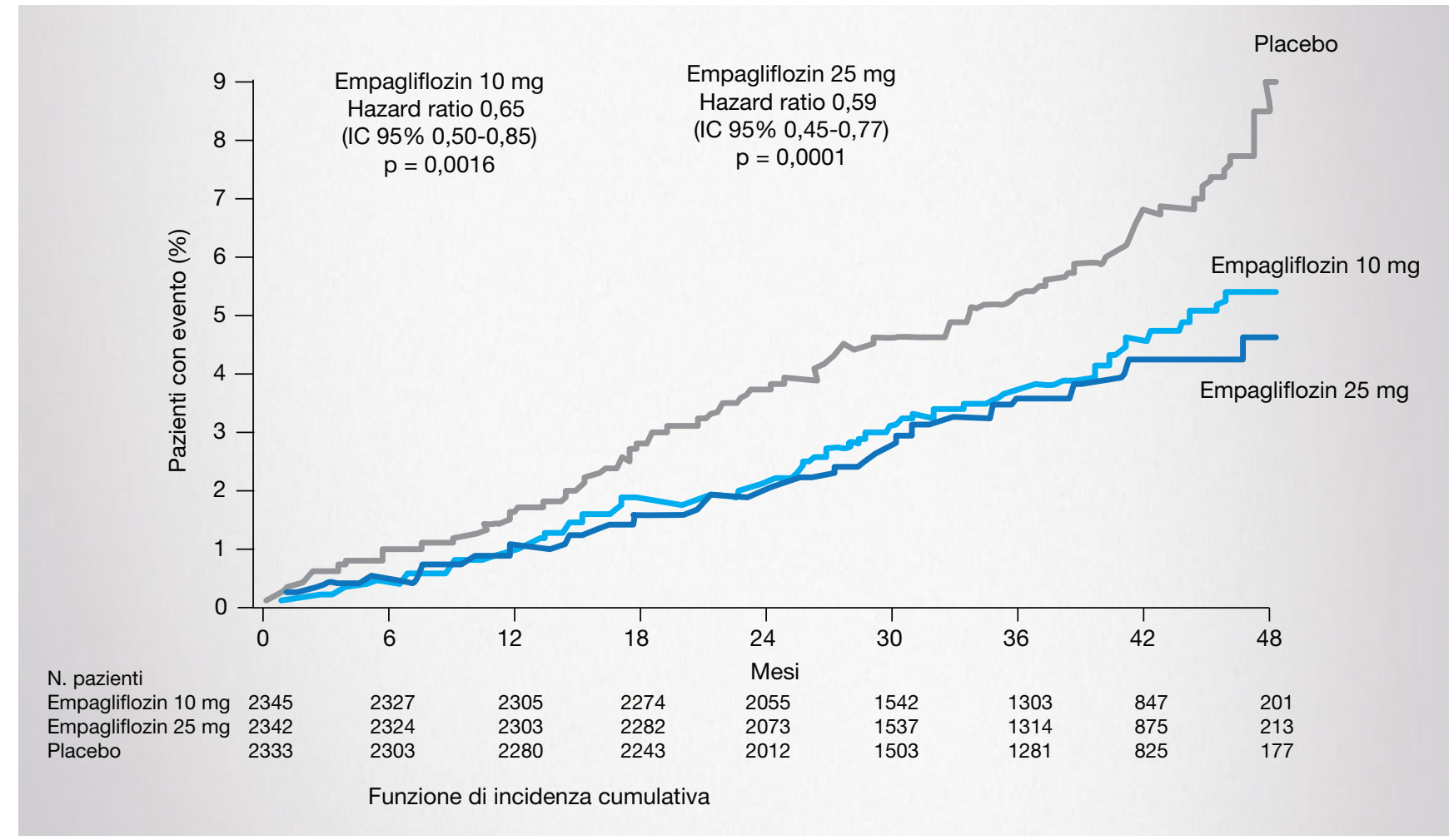

Figura 7. Mortalità cardiovascolare nei bracci dello studio EMPA-REG OUTCOME (modificata da [27]).

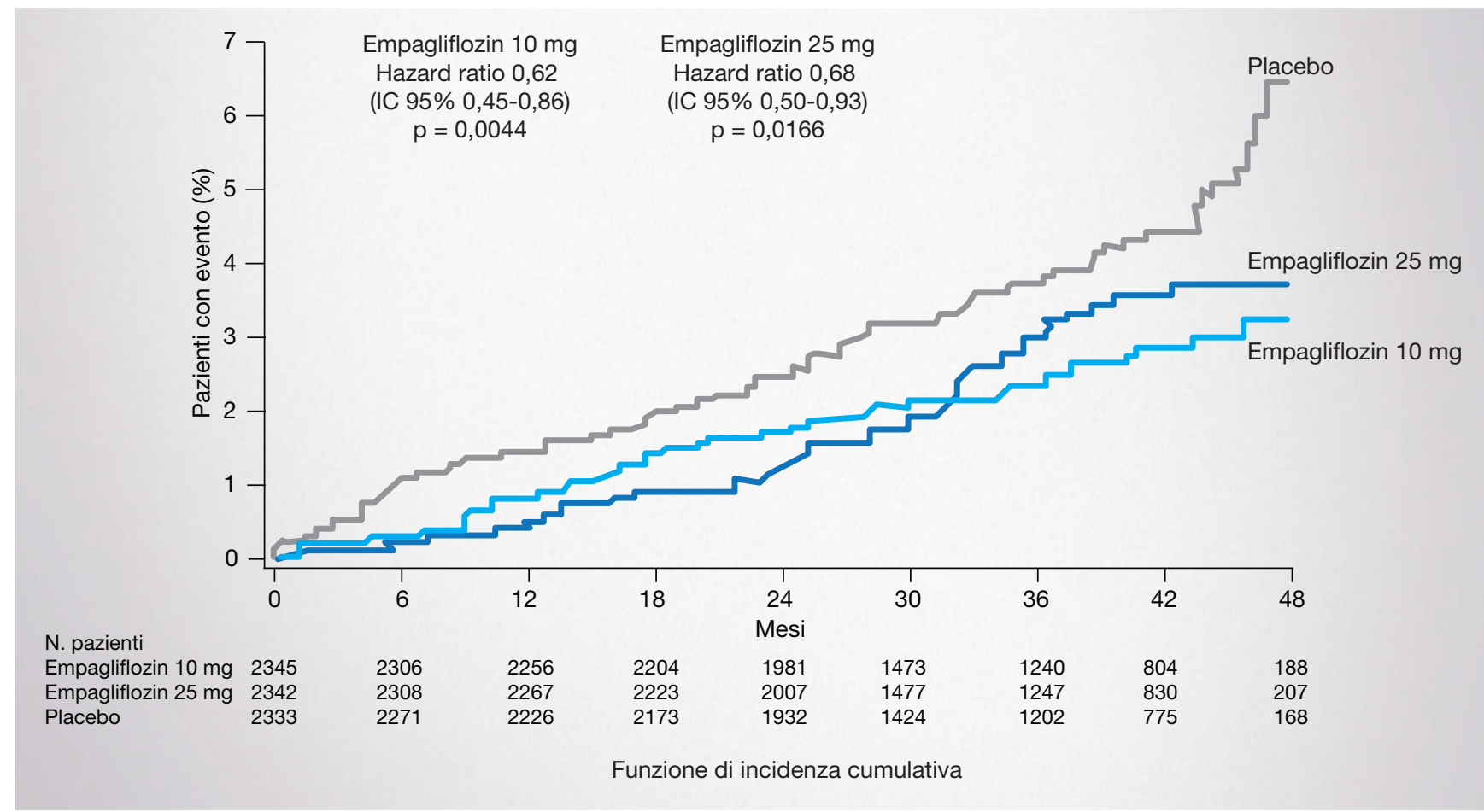

Figura 8. Ospedalizzazioni per scompenso cardiaco nello studio EMPA-REG OUTCOME (modificata da [27]). 
Tabella 4. Outcome degli studi LEADER ed EMPA-REG OUTCOME (modificata da [25,27]). CV, cardiovascolare; DMT2, diabete mellito di tipo 2; MACE, eventi cardiovascolari maggiori

\begin{tabular}{|c|c|c|}
\hline Outcome & $\begin{array}{c}\text { LEADER } \\
\text { Liraglutide } \\
9.340 \text { DMT2 ad alto rischio CV }\end{array}$ & \begin{tabular}{|c|} 
EMPA-REG OUTCOME \\
Empagliflozin \\
7.020 DMT2 con malattia CV
\end{tabular} \\
\hline 3 point MACE & $-13 \%$ & $-14 \%$ \\
\hline Morte CV & $-22 \%$ & $-38 \%$ \\
\hline Infarto miocardico fatale e non fatale & $\begin{array}{l}\quad-14 \% \\
\text { non fatale: }-12 \% \\
\text { non significativo }\end{array}$ & $\begin{array}{c}-13 \% \\
\text { non fatale: }-13 \% \\
\text { entrambi non significativi }\end{array}$ \\
\hline Ictus fatale e non fatale & $\begin{array}{c}-14 \% \\
\text { non fatale: }-11 \% \\
\text { entrambi non significativi }\end{array}$ & $\begin{array}{c}+18 \% \\
\text { non fatale: }+24 \% \\
\text { entrambi non significativi }\end{array}$ \\
\hline Ospedalizzazione per insufficienza cardiaca & $-13 \%$ non significativo & $-35 \%$ \\
\hline Mortalità per tutte le cause & $-15 \%$ & $-32 \%$ \\
\hline Nefropatia & $-22 \%$ & $-39 \%$ \\
\hline
\end{tabular}

$0,75-0,97 ; \mathrm{p}<0,001$ per la non-inferiorità; $\mathrm{p}=0,02$ per la superiorità) [30].

La mortalità per tutte le cause e la mortalità CV non sono risultate significative. La progressione di albuminuria era significativamente ridotta con canagliflozin rispetto al placebo (89,4 vs 128,7 pazienti per 1000/ anno), così come la regressione della microalbuminuria. Gli eventi avversi severi erano meno frequenti nei pazienti randomizzati a canagliflozin, ma il rischio di amputazioni agli arti inferiori era maggiore $(6,3$ vs 3,4 pazienti per 1000/anno; HR 1,97; IC 95\% 1,41-2,75).

Anche lo studio LEADER con liraglutide e SUSTAIN-6 con semaglutide in pazienti ad alto rischio CV hanno mostrato importanti benefici dei GLP1-RA nella prevenzione di eventi $\mathrm{CV}$.

Risulta quindi interessante mettere a confronto i risultati di questi studi dimostratisi di particolare beneficio sul versante CV. Dallo studio LEADER emerge che la riduzione dell'endpoint primario è pari al 13\%, rispetto al 14\% di EMPA-REG OUTCOME; tuttavia, confrontando gli altri parametri (mortalità per cause CV, ospedalizzazioni per scompenso cardiaco, mortalità per tutte le cause e nefropatia), i dati risultano diversi non raggiungendo ad esempio la significatività nella riduzione delle ospedalizzazioni per scompenso cardiaco. Nell'EMPA-REG OUTCOME è stato riscontrato un aumento di ictus, comunque non significativo
(Tabella 4). Va ricordato che nello studio LEADER le curve relative all'endpoint primario divergono tra braccio di controllo e farmaco in studio dopo 12-18 mesi dalla randomizzazione, evidenziando un probabile effetto di liraglutide sul processo aterosclerotico a differenza di empagliflozin, la cui rapidità di effetto sottende un meccanismo differente. Discorso analogo va fatto mettendo a confronto i risultati degli studi SUSTAIN-6 e CANVAS Program con quelli di EMPA-REG OUTCOME (Tabelle 5 e 6 ).

\section{Possibili meccanismi d'azione di empagliflozin}

Empagliflozin è un farmaco che non solo riduce la glicemia ma anche la pressione arteriosa, l'albuminuria e l'uricemia; migliora il profilo lipidico; diminuisce lo stress ossidativo e riduce il peso corporeo (Figura 9). Questi effetti non riescono tuttavia a spiegare la rapida divergenza delle curve osservata nello studio EMPA-REG OUTCOME in merito all'endpoint primario. È possibile che esistano recettori non noti a livello cardiaco, del sistema renina angiotensina-aldosterone e del sistema nervoso simpatico. È altresì possibile che a livello dei miocardiociti si ottenga una serie di effetti stabilizzanti di membrana e inotropi positivi. Probabilmente, un'importanza maggiore va attribuita al ruolo emodinamico con effetto ipovolemico 
Tabella 5. Outcome degli studi SUSTAIN-6 ed EMPA-REG OUTCOME (modificata da [26,27]). CV, cardiovascolare; DMT2, diabete mellito di tipo 2; MACE, eventi cardiovascolari maggiori

\begin{tabular}{|c|c|c|}
\hline Outcome & $\begin{array}{c}\text { SUSTAIN-6 } \\
\text { Semaglutide } \\
9.340 \text { DMT2 ad alto rischio CV }\end{array}$ & $\begin{array}{c}\text { EMPA-REG OUTCOME } \\
\text { Empagliflozin } \\
7.020 \text { DMT2 con malattia CV }\end{array}$ \\
\hline 3 point MACE & $-26 \%$ & $-14 \%$ \\
\hline Morte CV & $-2 \%$ non significativo & $-38 \%$ \\
\hline Infarto miocardico fatale e non fatale & $\begin{array}{l}\text { non fatale: }-26 \% \\
\text { non significativo }\end{array}$ & $\begin{array}{c}-13 \% \\
\text { non fatale: }-13 \% \\
\text { entrambi non significativi }\end{array}$ \\
\hline Ictus fatale e non fatale & non fatale: $-39 \%$ & $\begin{array}{c}+18 \% \\
\text { non fatale: }+24 \% \\
\text { entrambi non significativi }\end{array}$ \\
\hline Ospedalizzazione per insufficienza cardiaca & $+11 \%$ non significativo & $-35 \%$ \\
\hline Mortalità per tutte le cause & $+5 \%$ (non significativo) & $-32 \%$ \\
\hline Nefropatia & $-36 \%$ & $-39 \%$ \\
\hline
\end{tabular}

Tabella 6. Outcome degli studi CANVAS Program ed EMPA-REG OUTCOME (modificata da [27,30]). CV, cardiovascolare; DMT2, diabete mellito di tipo 2; MACE, eventi cardiovascolari maggiori

\begin{tabular}{|c|c|c|}
\hline Outcome & $\begin{array}{c}\text { CANVAS Program } \\
\text { Canagliflozin } \\
10.142 \text { DMT2 ad alto rischio CV }\end{array}$ & $\begin{array}{c}\text { EMPA-REG OUTCOME } \\
\text { Empagliflozin } \\
7.020 \text { DMT2 con malattia CV }\end{array}$ \\
\hline 3 point MACE & $-14 \%$ & $-14 \%$ \\
\hline Morte CV & $-13 \%$ non significativo & $-38 \%$ \\
\hline Infarto miocardico fatale e non fatale & $\begin{array}{c}-11 \% \\
\text { non fatale: }-15 \% \\
\text { entrambi non significativi }\end{array}$ & $\begin{array}{c}-13 \% \\
\text { non fatale: }-13 \% \\
\text { entrambi non significativi }\end{array}$ \\
\hline Ictus fatale e non fatale & $\begin{array}{c}-10 \% \\
\text { non fatale: }-11 \% \\
\text { entrambi non significativi }\end{array}$ & $\begin{array}{c}+18 \% \\
\text { non fatale: }+24 \% \\
\text { entrambi non significativi }\end{array}$ \\
\hline Ospedalizzazione per insufficienza cardiaca & $-33 \%$ & $-35 \%$ \\
\hline Mortalità per tutte le cause & $-13 \%$ non significativo & $-32 \%$ \\
\hline Nefropatia & $\begin{array}{c}\text { Progressione dell'albuminuria: } \\
\text {-37\%; Riduzione del } 40 \% \mathrm{di} \\
\text { eGFR, dialisi, o decesso da } \\
\text { nefropatia: }-40 \%\end{array}$ & $-39 \%$ \\
\hline
\end{tabular}

e antiaritmico (per ridotto stress di parete) che può spiegare la rapida ripercussione sugli outcome CV. Sodio e glucosio sono trasportati a livello del tratto tubulare renale S1 e S3 da due famiglie di cotrasportatori, gli SGLT2 e gli SGLT1. La prima famiglia si trova nel sistema prossimale del tubulo a livello S1 ed è responsabile del riassorbimento di circa l' $80 \%$ del glucosio dal lume tubulare, mentre la famiglia SGLT1 è più a valle ed è responsabile del riassorbimento di circa il 10-15\% del sodio e del glucosio. La capacità di riassorbimento è legata al valore di glicemia: al di sopra di $180 \mathrm{mg} / \mathrm{dl}$ il rene non è in grado di riassorbire tutto il glucosio a livello tubulare e quindi inizia a comparire glicosuria. Questo fe- 


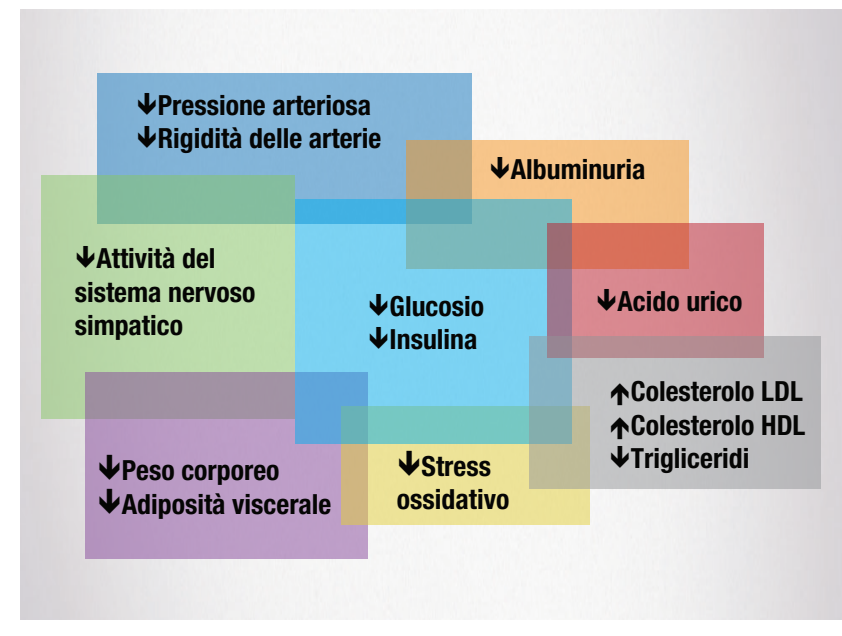

Figura 9. I molteplici e complessi effetti di empagliflozin (modificata da [31]).

nomeno, fino a poco tempo fa considerato negativo perché spia di un diabete scompensato, in realtà è stato rivalutato come fenomeno di compenso, tanto è vero che i nuovi farmaci sfruttano proprio questo tipo di meccanismo. Questi farmaci "sfruttano" questo meccanismo di tentativo di compenso e promuovono l'escrezione urinaria di glucosio: è possibile quindi che l'eliminazione del glucosio inneschi meccanismi di compenso energetico con utilizzo di acidi grassi a livello cardiaco, in modo da produrre effetti benefici.

\section{Trattamento del paziente ad alto rischio cardiovascolare: \\ il parere del mondo cardiologico}

Le osservazioni fin qui riportate impongono la necessità di fenotipizzare il più possibile il paziente diabetico: il paziente diabetico ad alto rischio $\mathrm{CV}$ va inquadrato come un soggetto da trattare con approccio intensivo non solo per quanto riguarda la glicemia ma anche i fattori di rischio $\mathrm{CV}$.

In un paziente anziano, con lunga durata di malattia, con comorbilità e pregressa malattia CV importante, l'intervento dovrà essere meno aggressivo rispetto a pazienti con quadro clinico meno compromesso. Ipercolesterolemia e ipertensione sono fattori di rischio CV che devono essere trattati specialmente nel diabetico ad alto rischio CV. I livelli di colesterolo LDL in que- sti pazienti dovrebbe essere idealmente $<70 \mathrm{mg} / \mathrm{dl}$ e i valori pressori $<140 / 80 \mathrm{mmHg}$.

In merito ai trattamenti ipoglicemizzanti esistono farmaci, quali le sulfaniluree (SU), che dovrebbero essere utilizzati con cautela e solo in specifiche situazioni. Da una metanalisi di studi osservazionali emerge come le SU aumentino il rischio di infarto miocardico dell'11\% rispetto ad altre terapie, dei MACE in generale dell'11\% e della mortalità CV del $26 \%$ [32]. La pericolosità delle SU da un punto di vista CV viene evidenziata anche da metanalisi di trial clinici randomizzati, da cui si rileva un aumento di mortalità per tutte le cause indotto da questi farmaci del 22\% [33]. Va sottolineato che in questi trial la proporzione di cardiopatici era del $20 \%$ : tale dato risulta ancora più preoccupante se proiettato nel diabetico a elevato rischio CV. La stessa metanalisi ha mostrato che le SU aumentano anche il rischio di ictus del 39\% rispetto agli altri ipoglicemizzanti, probabilmente a causa degli episodi ipoglicemici indotti da questo tipo di farmaci. Le SU comportano un rischio di ipoglicemia severa di dieci volte maggiore rispetto agli SGLT2 inibitori e ai DPP4 inibitori. Bisogna ricordare che l'intervallo di tempo fra il periodo trascorso in ipoglicemia e la comparsa di aritmie ventricolari è stretto. I DPP4 inibitori sono farmaci relativamente sicuri anche se dallo studio SAVOR-TIMI53 è emerso che saxagliptin può aumentare l'ospedalizzazione per scompenso cardiaco. Riteniamo pertanto che questo farmaco debba essere usato con cautela in pazienti con pregressa diagnosi di scompenso cardiaco [34]. Anche pioglitazone può aumentare significativamente il rischio di ospedalizzazione per scompenso cardiaco.

\section{Ricadute nella pratica clinica}

L'EMPA-REG OUTCOME, come gli altri CVOT, è uno studio che rispecchia la pratica clinica corrente: proprio per questo vengono definiti trial pragmatici. Quanto impatta dunque nella pratica clinica empagliflozin? Secondo lo studio EMPA-REG OUTCOME, il numero di pazienti ad alto rischio da trattare (NNT) per prevenire un decesso è simile a quanto osservato nello studio $4 \mathrm{~S}$ per simvastatina e nello 


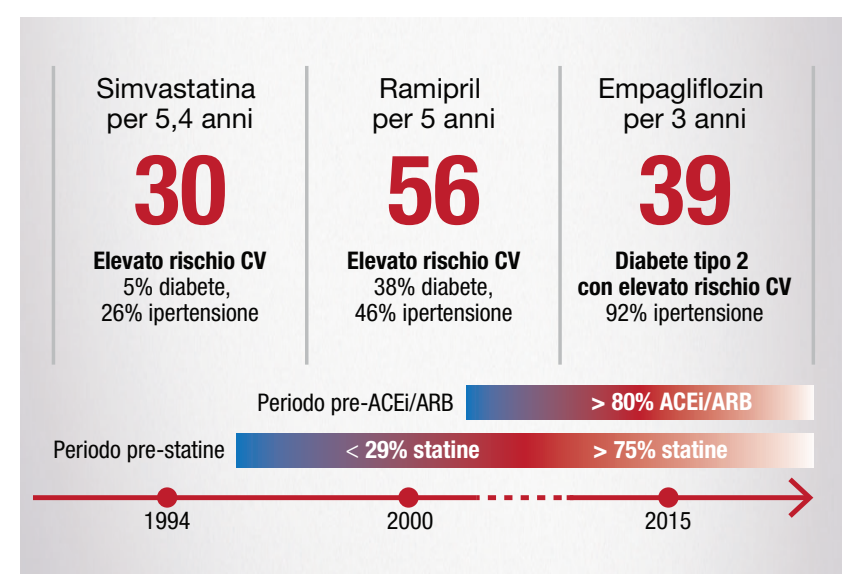

Figura 10. Numero di pazienti da trattare (NNT) per prevenire un decesso nei trial pivotali di riferimento in pazienti a elevato rischio cardiovascolare (modificata da [27,35,36]). ACEi, ACE inibitori; $\mathrm{ARB}$, antagonisti recettoriali dell'angiotensina II; CV, cardiovascolare

studio HOPE per ramipril, con la nota aggiuntiva che il valore per empagliflozin è stato ottenuto su pazienti ulteriormente trattati secondo i più moderni standard di terapia antipertensiva e ipolipemizzante (Figura 10).

In 1.000 pazienti diabetici ad alto rischio CV trattati per 3 anni, il trattamento con empagliflozin permette di salvare 25 vite ( 82 vs 57 morti) con 22 morti CV in meno (59 vs 37), comporta 14 ospedalizzazioni per scompenso in meno ( 42 vs 28), al prezzo di 53 infezioni genitali in più (22 vs 75).

Empagliflozin è stato inserito nelle linee guida canadesi, che lo raccomandano con grado A e livello $1 \mathrm{~A}$ nei pazienti con diabete di tipo 2 a elevato rischio CV con target glicemico non raggiunto [37]. Anche le linee guida ESC sullo scompenso cardiaco raccomandano (classe IIa, livello B) il farmaco per prevenire l'insufficienza cardiaca e prolungare la vita [38]. Nello studio EMPA-REG OUTCOME circa il $10 \%$ dei pazienti aveva una diagnosi di pregresso scompenso cardiaco al reclutamento, e gli outcome raggiunti in questo gruppo di pazienti sono risultati sovrapponibili al resto della popolazione [39]. Si può pertanto concludere che il farmaco è estremamente maneggevole e può essere associato ad altri ipoglicemizzanti e farmaci cardioattivi per la riduzione del rischio $\mathrm{CV}$.

\section{Nuove opportunità di nefroprotezione per il paziente con diabete di tipo 2}

Il diabete è la causa principale di insufficienza renale terminale (end-stage renal disease, ESRD), l'unica complicanza a lungo termine della malattia diabetica che negli ultimi anni non si è ridotta: nel 2009 in Italia, il diabete è stato causa del 19,6\% di nuovi casi di ESRD ed era secondo (25,3\%) solo all'ipertensione $(34,3 \%)$ quale condizione di comorbilità nei casi incidenti di ESRD [2]. In tutti i Paesi occidentalizzati, l'incidenza di nefropatia cronica e quella di ESRD sono in progressivo aumento nei pazienti con diabete mellito tipo 2 [40].

Nel diabete di tipo 2 il dato istologico è eterogeneo: solo un terzo dei soggetti con microalbuminuria e la metà di quelli con macroalbuminuria presentano un quadro di glomerulopatia diabetica tipica; nella maggior parte dei pazienti si repertano lesioni prevalentemente vascolari e/o tubulo-interstiziali o non è evidenziabile una patologia renale significativa. La microalbuminuria, ossia l'escrezione urinaria di albumina compresa tra 30 e $300 \mathrm{mg} / 24$ ore $(20-200 \mu \mathrm{g} /$ min) è il parametro più semplice e sensibile per rilevare il rischio di nefropatia nel diabete: nella forma di tipo 2 è stato riconosciuto, confermato e ridefinito il suo valore predittivo di nefropatia, ESRD e di morbilità e mortalità per cause CV [41,42]. Alla comparsa della microalbuminuria possono associarsi progressive alterazioni strutturali glomerulari, vascolari e tubulo-interstiziali, retinopatia diabetica, aumento della pressione arteriosa, sfavorevole profilo lipidico (sindrome metabolica), attivazione del processo infiammatorio, disfunzione e attivazione endoteliale, insulino-resistenza, che preludono alla progressione verso la proteinuria e la riduzione del filtrato $[43,44]$. Nel diabete mellito di tipo 2, la prevalenza di microalbuminuria è di circa il 20\%; la microalbuminuria e talvolta anche la nefropatia conclamata possono essere presenti già alla diagnosi (fino al 10-15\% dei soggetti). La microalbuminuria può:

- regredire,

- rimanere a lungo nel range che la identifica,

- progredire. 
La sua progressione, in media del $10-15 \%$ per anno, evolve verso la macroalbuminuria (albuminuria $>300 \mathrm{mg} / 24$ ore o $>200 \mu \mathrm{g} / \mathrm{min}$; proteinuria $>500$ $\mathrm{mg} / 24$ ore) e, talora, verso la sindrome nefrosica (proteinuria $>3,5 \mathrm{~g} / 24$ ore). Al comparire di macroalbuminuria, ma già in presenza di microalbuminuria, il filtrato glomerulare tende a ridursi progressivamente [41].

Nel paziente con nefropatia diabetica è raccomandato un approccio multifattoriale per il raggiungimento dei target di glicemia, pressione arteriosa e lipidemia. Il controllo intensivo del glucosio riduce il rischio di macroalbuminuria [45] ed è associato a una significativa diminuzione del rischio di ESRD [46].

Il progressivo peggioramento della funzionalità renale nel diabetico di tipo 2 si associa a un progressivo aumento della mortalità per cause CV. I dati dello Swedish National Diabetes Register relativi a 435.369 pazienti con diabete di tipo 2 e 2.117 .483 controlli evidenziano chiaramente come il passaggio da una condizione di normoalbuminuria, a microalbuminu- ria, macroalbuminuria ed ESRD aumenti di molto il rischio di eventi CV nella popolazione diabetica [47] (Figura 11); in questo tipo di pazienti quindi è particolarmente utile utilizzare dei farmaci che abbiano la capacità di ridurre il rischio $\mathrm{CV}$.

Lo studio EMPA-REG OUTCOME ha dimostrato che empagliflozin agisce su tutti gli outcome renali: rallenta la progressione di malattia renale che normalmente si riscontra nel diabetico preservando il filtrato glomerulare, riduce lo sviluppo di albuminuria, rallenta il raddoppio della creatinina sierica e la necessità di ricorrere al trattamento dialitico [48] (Figura 12).

Nello studio EMPA-REG OUTCOME non è stata osservata un'interazione significativa tra valori di filtrato glomerulare, 3 point MACE e mortalità $\mathrm{CV}$, quindi, indipendentemente dallo stato di funzione renale, empagliflozin determina risultati positivi in termini di riduzione di eventi CV (Figura 13 [49]).

Nei pazienti con filtrato glomerulare $<60 \mathrm{ml} / \mathrm{min}$, quindi portatori di malattia renale cronica stadio III,

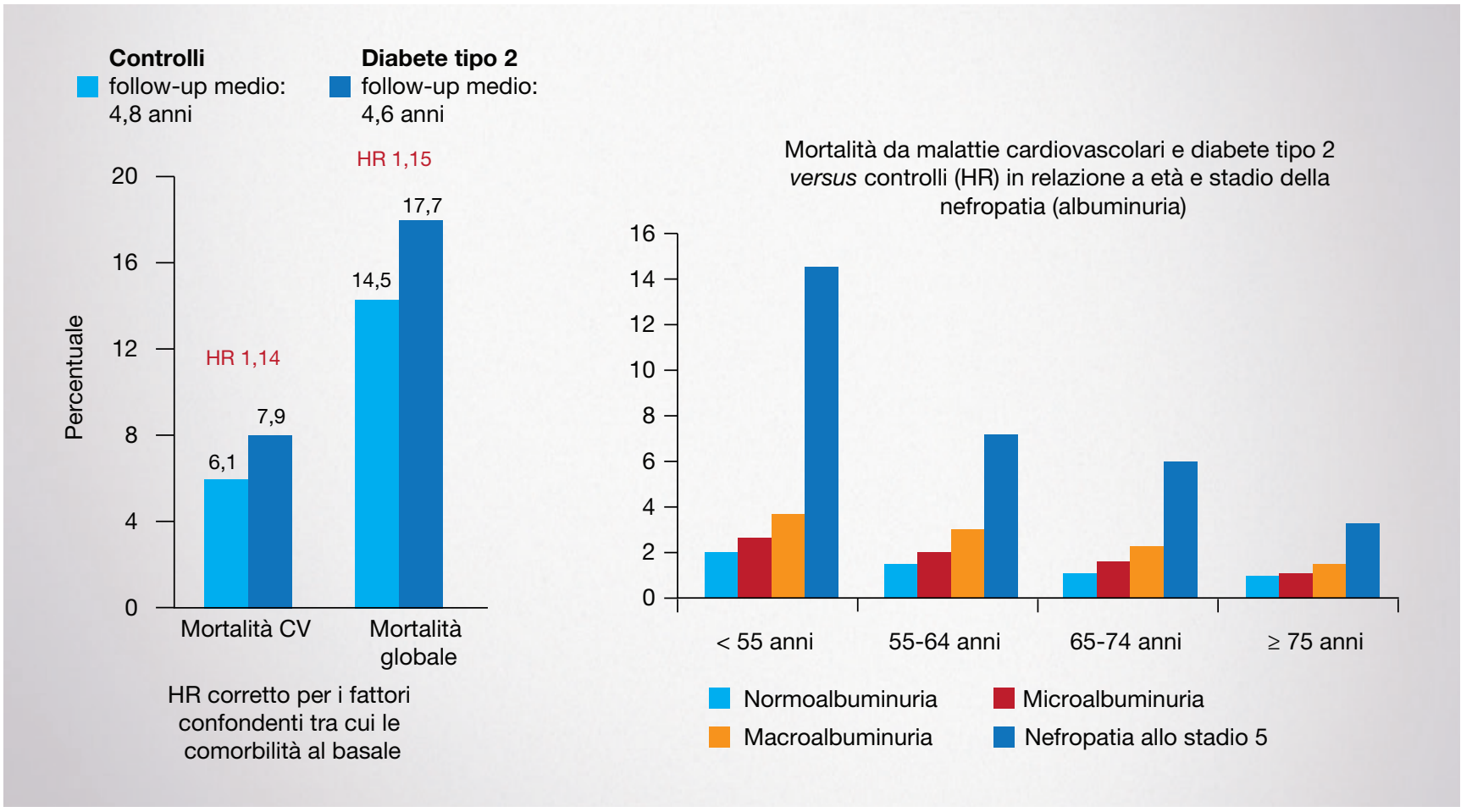

Figura 11. Swedish National Diabetes Register. correlazione tra peggioramento della funzionalità renale e mortalità cardiovascolare nei diabetici di tipo 2 (modificata da [47]). CV, cardiovascolare; HR, hazard ratio 


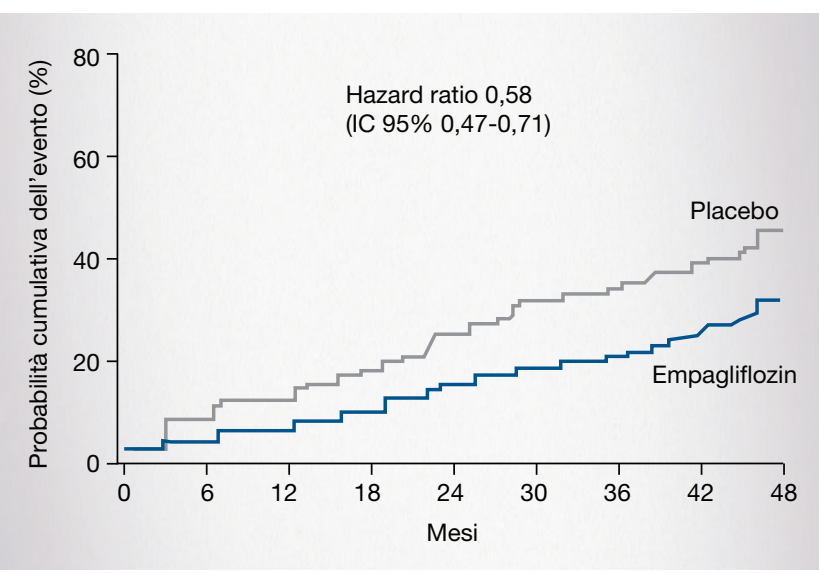

Figura 12. Studio EMPA-REG OUTCOME: effetto sulla nefropatia (nuovi casi o peggioramento) osservato nei gruppi di trattamento con empagliflozin (modificata da [27]).

le ospedalizzazioni per scompenso cardiaco si riducono del 51\% nei pazienti trattati con empagliflozin. L'SGLT2 inibitore riduce la pressione glomerulare e quindi induce, inizialmente, un lieve temporaneo decremento del filtrato glomerulare, espressione dell'effetto del farmaco, che nel tempo si normalizza. Bisogna considerare inoltre che l'effetto di riduzione della pressione sistolica, di modulazione del sistema renina-angiotensina-aldosterone, di perdita di peso corporeo sono elementi in grado di per sé di ridurre il filtrato; tuttavia i dati dello studio mostrano il miglioramento della funzionalità renale con inversione delle curve relative all'andamento dell'eGFR dopo i primi mesi di terapia (Figura 14).

È comunque opportuno ricordare che, nel rispetto del Riassunto delle Caratteristiche del Prodotto, empagliflozin non può essere utilizzato in pazienti con eGFR $<60 \mathrm{ml} / \mathrm{min}$; tuttavia può essere continuato fino a valori di eGFR di $45 \mathrm{ml} / \mathrm{min}$ qualora il paziente abbia iniziato la terapia con valori di eGFR $\geq 60 \mathrm{ml} /$ $\min [29]$

\section{Insulina glargine: quale ruolo nella terapia del diabete tipo 2}

Insulina glargine $100 \mathrm{U} / \mathrm{ml}$ ha permesso di ottenere una insulinizzazione basale vera e fisiologica, conseguendo non solo una durata d'azione prolungata ma anche un ridotto rischio di episodi ipoglicemici notturni.

Dal punto di vista dell'efficacia, nel Treat-to-tar-

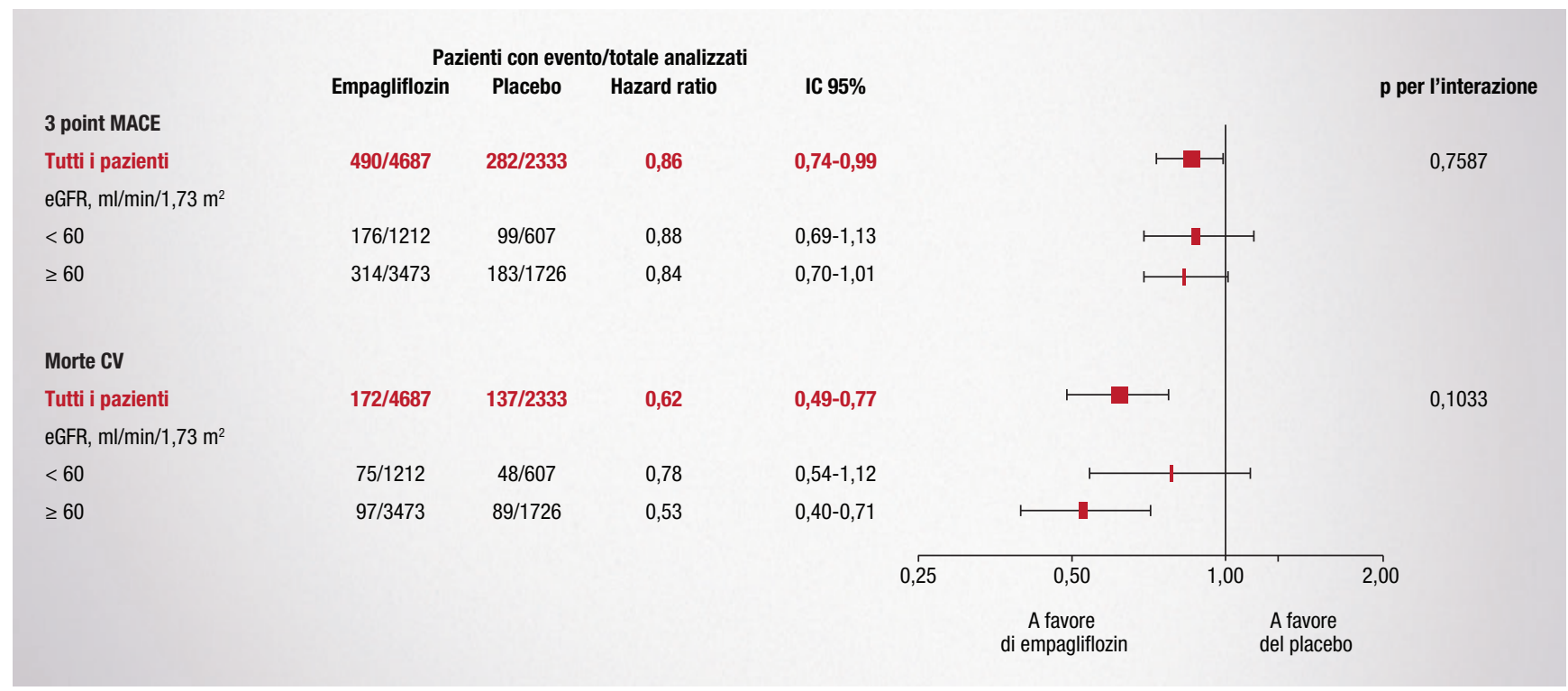

Figura 13. Studio EMPA-REG OUTCOME: 3 point MACE e morte cardiovascolare (CV) in funzione dei livelli di filtrato glomerulare stimato (eGFR) (modificata da [49]). MACE, evento cardiovascolare maggiore; 3 point MACE è il tempo al primo evento di morte CV, infarto miocardico non fatale 0 ictus non fatale 


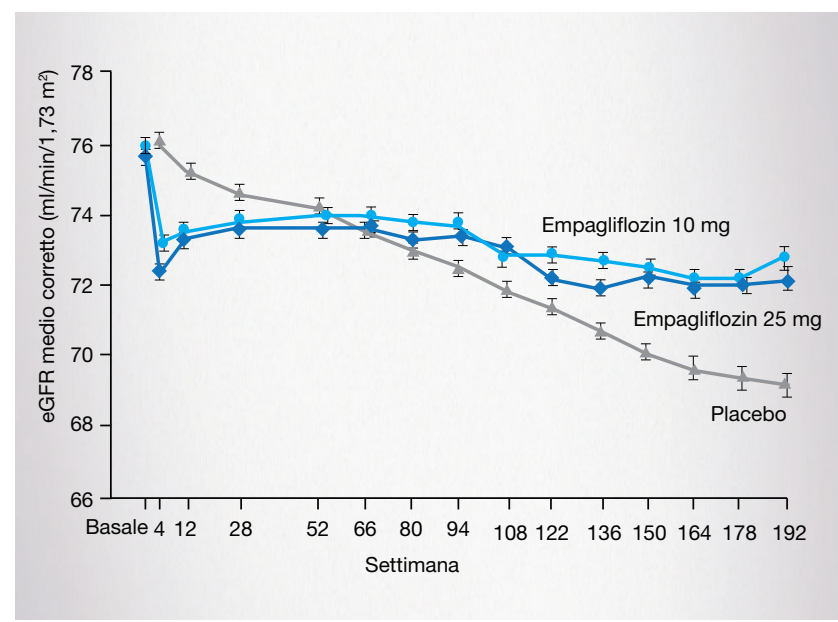

Figura 14. Andamento del filtrato glomerulare stimato (eGFR) nei tre bracci dello studio EMPA-REG OUTCOME (modificata da [48]).

get trial non è stata dimostrata alcuna differenza nel controllo glicemico (Figura 15) e nell'aumento di peso tra insulina glargine $100 \mathrm{U} / \mathrm{ml}$ e insulina NPH [50].

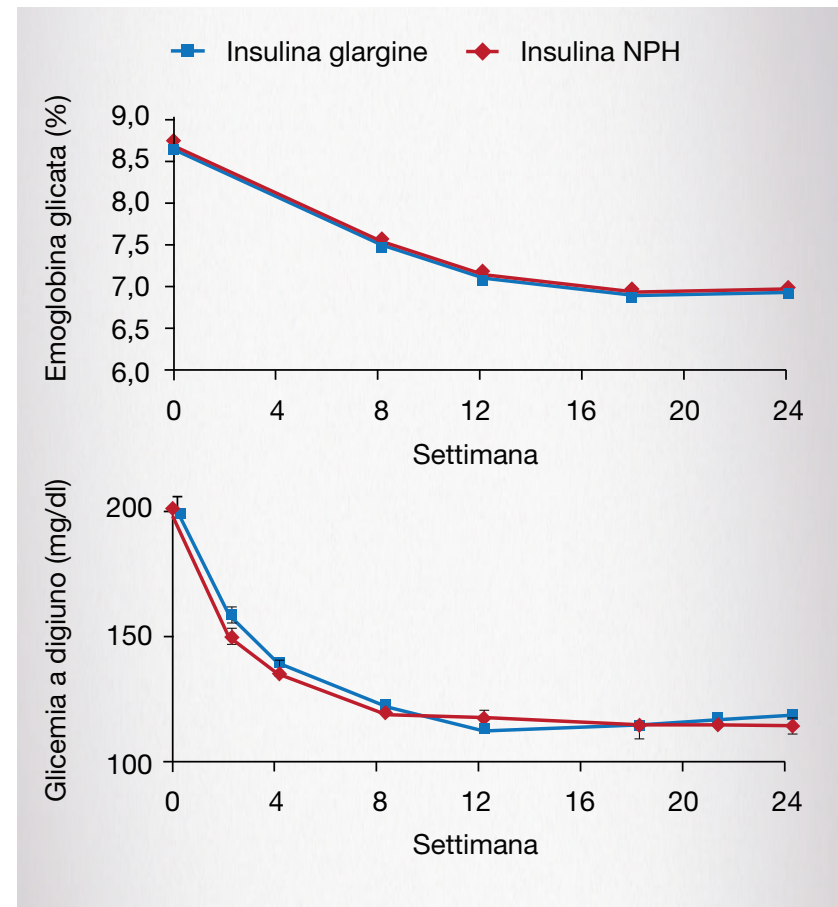

Figura 15. Treat-to-target trial: confronto del controllo glicemico (emoglobina glicata e glicemia a digiuno) tra i due tipi di insulina (modificata da [50]).

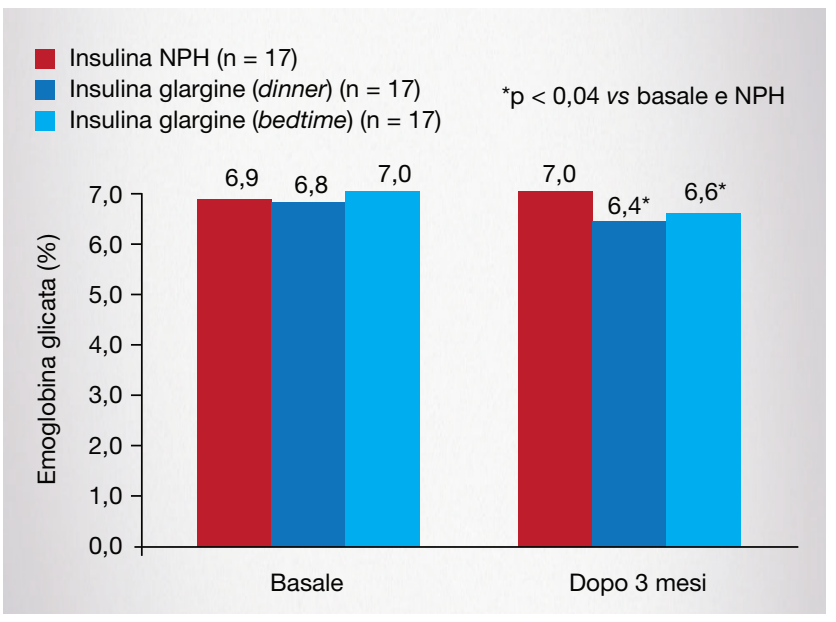

Figura 16. Controllo glicemico con insulina glargine $100 \mathrm{U} / \mathrm{ml}$ e NPH nei pazienti diabetici di tipo 1 (modificata da [51]).

Analogamente, per quanto riguarda i miglioramenti dell'HbA1c, l'effetto di glargine $100 \mathrm{U} / \mathrm{ml}$ si è dimostrato sovrapponibile sia a quello di NPH sia di detemir.

Anche nel diabete di tipo 1, la riduzione dell'HbA1c è risultata sovrapponibile tra glargine e NPH, ma con un controllo significativamente migliore della glicemia a digiuno con glargine $100 \mathrm{U} / \mathrm{ml}$ (Figura 16) [51].

Glargine $100 \mathrm{U} / \mathrm{ml}$ si è dimostrata efficace anche nei pazienti pediatrici con diabete di tipo 1 , in cui, a differenza di NPH, non ha richiesto un aumento del dosaggio. Anche nella popolazione anziana suddivisa per 3 sottogruppi di età $(<65$ anni, tra 65 e 74 anni, $>74$ anni), si è avuta un'ottima riduzione della glicemia a digiuno e dell'HbA1c. È interessante notare che tra i tre sottogruppi, la dose totale giornaliera di insulina è risultata più bassa nella popolazione più anziana (> 74 anni).

\section{Ipoglicemie e rischio cardiovascolare}

L'ipoglicemia è un evento problematico che deve essere evitato poiché non solo può portare a morte ma anche associarsi a eventi cardiovascolari maggiori. Dai dati degli studi Treat-to-target e LANMET [52], emerge che insulina glargine $100 \mathrm{U} / \mathrm{ml}$ induce un numero di episodi ipoglicemici sia sintomatici che notturni significativamente inferiore rispetto a NPH (Figura 17). 


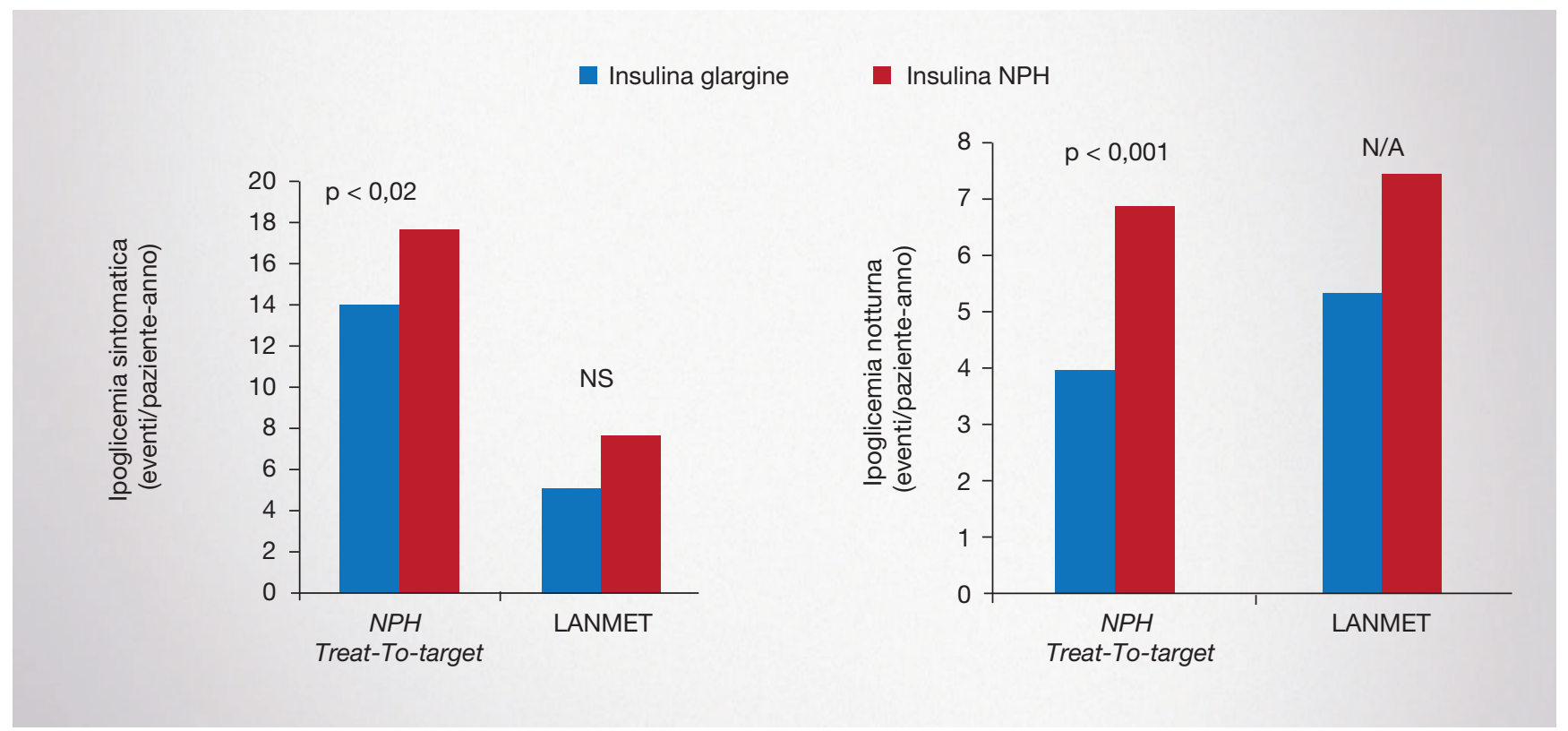

Figura 17. Tassi di eventi ipoglicemici negli studi Treat-to-targete LANMET (modificata da [52]). N/A, non applicabile; NS, non significativo

Anche nei pazienti di tipo 1, glargine causa un'incidenza di ipoglicemie significativamente inferiore rispetto a NPH, con una differenza che risulta essere anche maggiore rispetto a quella osservata nei pazienti di tipo 2 [50].

Per quanto riguarda la valutazione del possibile rischio CV associato all'utilizzo di glargine $100 \mathrm{U} / \mathrm{ml}$, è stato condotto lo studio ORIGIN della durata di 6 anni, su oltre 12.000 pazienti con diabete franco di tipo 2 o con ridotta tolleranza ai carboidrati, al fine di dimostrare l'impatto dell'uso di insulina basale sugli outcome CV (mortalità, infarto non fatale e ictus non fatale come endpoint primari) [53]. Rispetto al trattamento standard, insulina glargine $100 \mathrm{U} / \mathrm{ml}$ non solo ha dimostrato neutralità $\mathrm{CV}$, ma anche un effetto protettivo se si considerano l'ospedalizzazione per scompenso cardiaco o le amputazioni (Figura 18).

Lo studio ORIGIN ha dimostrato, inoltre, che i pazienti trattati con glargine $100 \mathrm{U} / \mathrm{ml}$ non presentavano differenze nell'incidenza di cancro o di morte per cancro rispetto ai pazienti in trattamento standard: ciò suggerisce che è la patologia diabetica in sé e non l'uso di questa insulina che espone il paziente a un rischio maggiore di patologia tumorale.

\section{Efficacia e sicurezza di glargine biosimilare}

La realtà dei biosimilari è presente da oltre 10 anni nella pratica clinica di altre specialità, come per esempio l'endocrinologia con i biosimilari del GH e la nefrologia con i biosimilari dell'eritropoietina. Nel campo specifico del diabete rappresentano invece una relativa novità rendendosi innanzitutto necessario chiarire alcuni concetti di base come la differenza tra generico e biosimilare. È fondamentale specificare che la sequenza aminoacidica del biosimilare è identica a quella dell'originator, ma che sono le eventuali differenze nel processo produttivo che possono determinare minime variazioni nel ripiegamento della molecola e quindi nella sua attività biologica. Queste differenze minimali vanno però considerate nell'ambito del processo produttivo tanto del biosimilare quanto dell'originator stesso, in quanto tipiche di tutti i prodotti biologici.

Gli studi ELEMENT 1 e 2 hanno dimostrato la sovrapponibilità di efficacia e sicurezza (emoglobina glicata, controllo glicemico, episodi ipoglicemici, peso, dosaggio insulinico) tra insulina glargine $100 \mathrm{U} / \mathrm{ml}$ originator e il suo biosimilare, sia nei pazienti di tipo 1 che di tipo 2 [54,55] (Figure 19 e 20).

Per quanto riguarda gli eventi allergici e le reazioni 


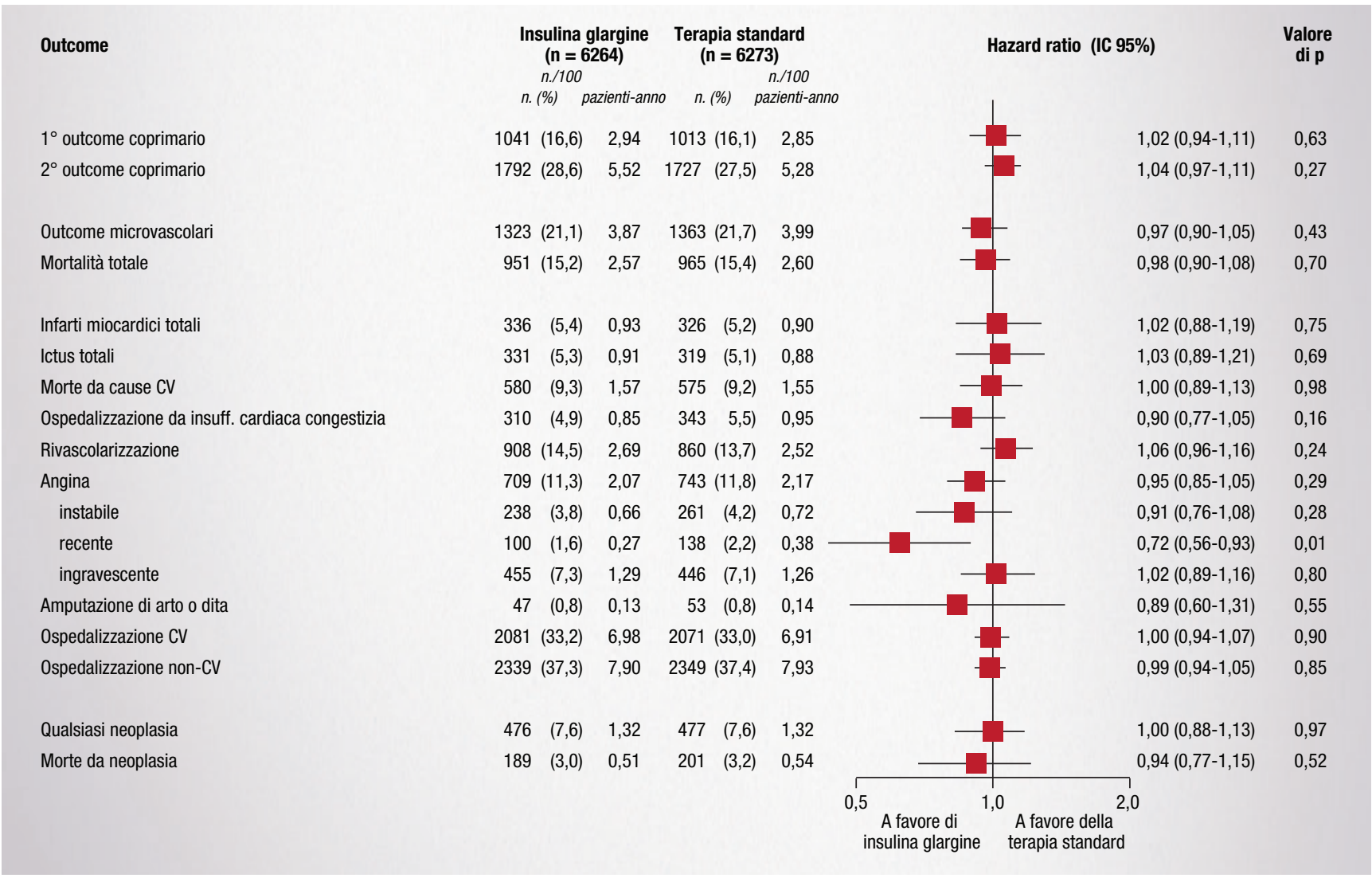

Figura 18. Riduzione degli eventi cardiovascolari con insulina glargine $100 \mathrm{U} / \mathrm{ml}$ osservati nello studio ORIGIN (modificata da [53]). CV, cardiovascolare
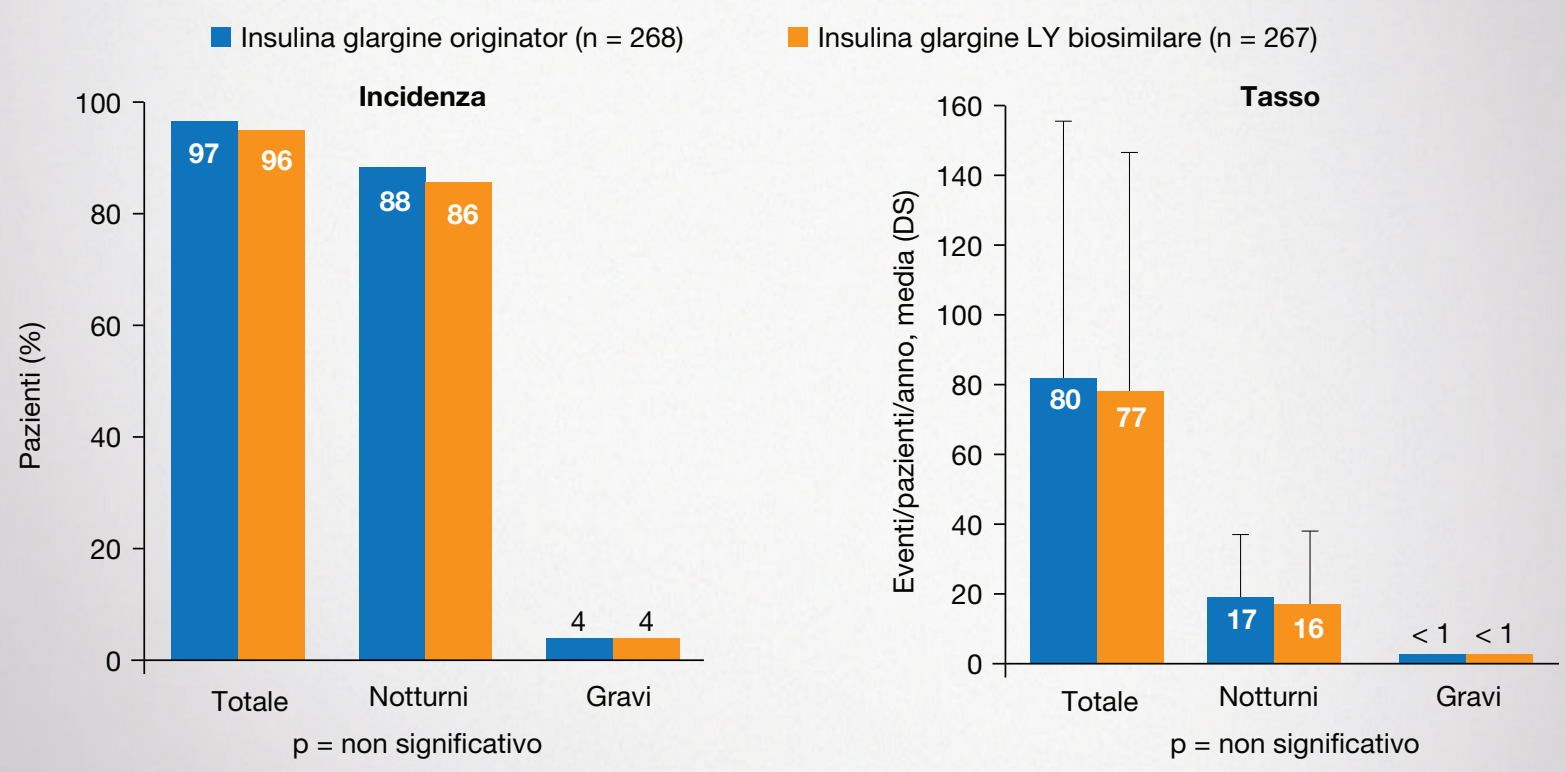

Figura 19. Studio ELEMENT 1: episodi ipoglicemici totali, notturni e gravi (modificata da [54]). 


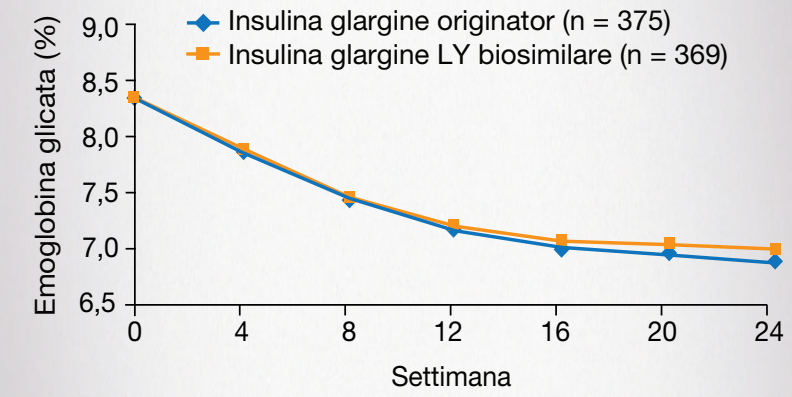

Figura 20. Studio ELEMENT 2: variazioni dell'emoglobina glicata nel tempo (modificata da [55]).

nel sito di iniezione, non è stata osservata alcuna differenza significativa tra le due molecole.

Uno degli aspetti da valutare con l'uso dei biosimilari è la possibile immunogenicità e a questo scopo sono state condotte analisi specifiche: sia nello studio ELEMENT 1 che nello studio ELEMENT 2 non sono state riportate differenze significative nella produzione di anticorpi anti-insulina tra glargine originator e biosimilare.

\section{Conclusioni}

Il diabete mellito è una malattia cronica complessa, che richiede frequenti e diversificati interventi sui livelli glicemici e sui fattori di rischio CV volti a prevenire le complicanze acute e croniche della malattia. Un'attività educativa della persona diabetica, finalizzata all'acquisizione delle nozioni e delle competenze necessarie all'autogestione della malattia, è un elemento di primaria importanza sia per il controllo glicemico sia per la prevenzione delle complicanze stesse.

L'introduzione di nuovi trattamenti, in particolare di nuovi farmaci (SGLT2 inibitori, alcuni analoghi dell'insulina, alcuni nuovi GLP1 agonisti) e di nuove tecnologie (modalità innovative di monitoraggio della glicemia, sistemi integrati microinfusori-sensore) ha modificato in modo sostanziale l'approccio terapeutico e impone al medico una visione più allargata della malattia diabetica in cui le parole d'ordine di- ventano con sempre maggiore evidenza "multidisciplinarietà" e "personalizzazione" secondo il concetto di patient centered medicine proposto dall'American Diabetes Association.

Tale necessità nasce da considerazioni di ordine epidemiologico, sociale e farmacoeconomico.

Il diabete è decisamente una patologia "sociale", dal momento che per la sua elevata prevalenza coinvolge di fatto gran parte della popolazione (pazienti e loro familiari). Ogni minuto, nel nostro Paese viene effettuata una nuova diagnosi di diabete; ogni 3 minuti e mezzo un diabetico ha un attacco cardiaco, ogni 10 minuti un diabetico muore. Nonostante tutto quanto scritto sia impressionante, la consapevolezza della malattia diabetica in Italia non è ancora così diffusa. Una malattia con tale prevalenza non può non impattare fortemente anche sulla spesa: i costi del diabete per il budget della sanità italiana ammontano al 9\% delle risorse. Questo significa più di 9,22 miliardi di euro all'anno o 1,05 milioni di euro ogni ora. In media, ogni malato spende 2.600 euro l'anno per la sua salute, più del doppio rispetto ai concittadini senza diabete, incidendo per il 5,61\% sulla spesa sanitaria e per lo 0,29\% sul PIL [56].

È da sottolineare che di questi 2.600 euro solo il $7 \%$ deriva dalla spesa per i farmaci, mentre il 25\% è legato ai costi delle complicanze cardiovascolari, renali, oculari e neuropatiche (si calcola che il costo per i pazienti che presentano complicanze sia addirittura quadruplo rispetto ai pazienti senza complicanze) e una fetta ancora più cospicua è relativa alle ospedalizzazioni per diabete (circa il 20\% delle persone ospedalizzate sono affette da questa patologia). Tutto questo senza considerare i costi intangibili (coinvolgimento di familiari e difficoltà nelle relazioni sociali) e quelli legati alla mancata produttività.

Alla sfida posta da questa malattia occorre quindi dare una risposta forte, che veda impegnati i medici, gli operatori sanitari, le istituzioni, la società e i cittadini oltre ovviamente ai pazienti affetti e alle loro famiglie: la risposta forte consiste nella definizione di azioni da intraprendere e nell'individuazione di modelli organizzativi nel quale queste azioni vengano collocate. Non c'è quindi dubbio che un ruolo cruciale sia svol- 
to dalla gestione clinica della malattia, un ruolo che impone l'acquisizione delle novità scientifiche da integrare nel proprio bagaglio di conoscenze tecniche. Quanto discusso nel presente documento ha permesso di portare all'attenzione dei clinici come i più recenti trial di outcome cardiovascolare abbiano ampliato le conoscenze sugli agenti ipoglicemizzanti in merito alla prevenzione del rischio cardiocircolatorio. In particolare, gli studi EMPA-REG OUTCOME e LEADER hanno fornito evidenze robuste su come farmaci innovativi, quali gli SGLT2 inibitori e i GLP1 agonisti, possano assicurare al paziente diabetico una protezione efficace e durevole da eventi cardiovascolari maggiori. Colpisce in particolare l'effetto di protezione dalla mortalità, sia cardiovascolare sia totale, offerto da empagliflozin nel diabetico ad alto rischio.

Va ricordato e sottolineato che gli eventi cardiovascolari sono la prima causa di morte nel paziente

\section{Disclosures}

Financial support: questa pubblicazione è stata resa possibile grazie a un grant non condizionante di Boheringer Ingelheim ed Eli Lilly.

Conflict of interest: il prof. Angelo Avogaro certifica che tutto il supporto materiale e finanziario per questa ricerca e per questo lavoro è chiaramente identificato di seguito: Novo Nordisk; Amgen; Astrazeneca; BoehringherIngelheim; Servier; Lilly; Sanofi; Takeda; Mediolanum; Merck Sharp \& Dohme; Janssen; Menarini Diagnostici; Novartis; Bruno Farmaceutici.

Il prof. Antonio Bossi certifica che tutto il supporto materiale e finanziario per questa ricerca e per questo lavoro è chiaramente identificato di seguito. Nei 5 anni passati ho ricevuto consultancies, honoraria o grants da: Lilly Italia SpA; Novo Nordisk Italia SpA; Boehringer Ingelheim Italia SpA; Bayer SA; Takeda Italia SpA; Artsana SpA; Sanofi Italia SpA; Johnson \& Johnson Italia SpA.

\section{Ringraziamenti}

Gli autori ringraziano:

- Paolo Calabrò, Divisione di Cardiologia, II Università di Napoli, Napoli

- Pasquale Perrone Filardi, Università Federico II, Napoli

- Andrea Giaccari, UO Endocrinologia e Metabolismo, Università Cattolica del Sacro Cuore, Policlinico Gemelli, Roma

- Matteo Monami, Direttore Unit Piede Diabetico, SOD Diabetologia, AOU Careggi, Firenze

- Gianluca Perseghin, Professore Ordinario di Endocrinologia Dipartimento di Medicina e Chirurgia - Università degli Studi di Milano Bicocca; Direttore Dipartimento di Medicina e Riabilitazione \& Re- con diabete di tipo 2 e che ogni terapia che sia in grado di ridurre significativamente questi eventi può migliorare la qualità e la quantità di vita di questi pazienti. Oggi, grazie alle nuove terapie a disposizione, si può probabilmente smettere di fare una "cosmesi" della glicemia e andare, invece, al cuore del problema che è quello di impattare sul profilo di rischio del paziente.

Il trattamento deve prevedere un approccio multifattoriale complessivo, che non può e non deve limitarsi al momento del ricovero ospedaliero per un evento cardiovascolare ma proseguire anche efficacemente nel follow-up del paziente. È quindi auspicabile un rapporto più stretto tra l'ospedale e il territorio, con network che coinvolgano il cardiologo, il diabetologo e il medico di medicina generale per una gestione ottimale dei pazienti e a beneficio del Sistema Sanitario Nazionale.

sponsabile Unità di Medicina Metabolica Policlinico di Monza, Monza

- Giuseppe Penno, UO di Malattie Metaboliche e Diabetologia, AOU Pisana, Pisa

- Roberto Trevisan, Direttore USC Malattie Endocrine-Diabetologia, AO Papa Giovanni XXIII, Bergamo

- Franco Arturi, Medicina Interna, AOU Mater Domini, Catanzaro

\section{Bibliografia}

1. Radke PW, Schunkert H. Diabetics with acute coronary syndrome: advances, challenges, and uncertainties. Eur Heart J 2010;31:2971-3.

2. SIN-RIDT Report 2011. Report Referring to the Calendar Year 2009. Consultabile su http://www.sin-ridt.org/sin-ridt.org.htm

3. Paneni F, Beckman JA, Creager MA, Cosentino F. Diabetes and vascular disease: pathophysiology, clinical consequences, and medical therapy: part I. Eur Heart J 2013;34(31):2436-43.

4. Ceriello A. New insights on oxidative stress and diabetic complications may lead to a "causal" antioxidant therapy. Diabetes Care 2003 May;26(5):1589-96

5. Wronkowitz N, Görgens SW, Romacho T et al. Soluble DPP4 induces inflammation and proliferation of human smooth muscle cells via protease-activated receptor 2. Biochim Biophys Acta 2014 Sep;1842(9):1613-21.

6. Fadini GP, Menegazzo L, Scattolini V et al. A perspective on NETosis in diabetes and cardiometabolic disorders. Nutr Metab Cardiovasc Dis 2016 Jan;26(1):1-8. doi: 10,1016/j.numecd.2015.11.008. Epub 2015 Nov 25 
7. Rigato M, Avogaro A, Fadini GP. Levels of circulating progenitor cells, cardiovascular outcomes and death: a meta-analysis of prospective observational studies. Circ Res 2016 Jun 10;118(12):1930-9.

8. Emerging Risk Factors Collaboration; Di Angelantonio E, Gao P, Khan $\mathrm{H}$ et al. Glycated hemoglobin measurement and prediction of cardiovascular disease. JAMA 2014 Mar 26;311(12):1225-33.

9. Authors/Task Force Members, Rydén L, Grant PJ, Anker SD et al. ESC Guidelines on diabetes, pre-diabetes, and cardiovascular diseases developed in collaboration with the EASD: the Task Force on diabetes, pre-diabetes, and cardiovascular diseases of the European Society of Cardiology (ESC) and developed in collaboration with the European Association for the Study of Diabetes (EASD). Eur Heart J 2013;34(39):3035-87.

10. Wang P, Huang R, Lu S et al. HbA1c below $7 \%$ as the goal of glucose control fails to maximize the cardiovascular benefits: a meta-analysis. Cardiovasc Diabetol 2015;14:124.

11. Boussageon R, Bejan-Angoulvant T, Saadatian-Elahi M et al. Effect of intensive glucose lowering treatment on all cause mortality, cardiovascular death, and microvascular events in type 2 diabetes: meta-analysis of randomised controlled trials. BMJ 2011;343:d4169.

12. Gaede $\mathrm{P}$, Vedel $\mathrm{P}$, Larsen $\mathrm{N}$ et al. Multifactorial intervention and cardiovascular disease in patients with type 2 diabetes. $\mathrm{N}$ Engl J Med 2003;348(5):383-93.

13. Brownrigg JR, Hughes CO, Burleigh D et al. Microvascular disease and risk of cardiovascular events among individuals with type 2 diabetes: a population-level cohort study. Lancet Diabetes Endocrinol 2016;4(7):588-97.

14. Emerging Risk Factors Collaboration; Di Angelantonio E, Kaptoge S, Wormser D et al. Association of cardiometabolic multimorbidity with mortality. JAMA 2015 Jul 7;314(1):52-60

15. AMD-SID - Standard italiani per la cura del diabete mellito 2016. Consultabile su http://www.standarditaliani.it/skin/www. standarditaliani.it/pdf/STANDARD_2016_June20.pdf Ultimo accesso: 31 gennaio 2017.

16. Boussageon R, Gueyffier F, Cornu C. Metformin as firstline treatment for type 2 diabetes: are we sure? BMJ 2016;352:h6748

17. ADVANCE Collaborative Group; Patel M, MacMahon S et al. Intensive blood glucose control and vascular outcomes in patients with type 2 diabetes. N Engl J Med 2008;358(24):2560-72.

18. Chiasson JL, Josse RG, Gomis R et al.; STOP-NIDDM Trial Research Group. Acarbose treatment and the risk of cardiovascular disease and hypertension in patients with impaired glucose tolerance: the STOP-NIDDM trial. JAMA 2003;290(4):486-94.

19. Van de Laar FA, Lucassen PL, Akkermans RP et al. Alphaglucosidase inhibitors for type 2 diabetes mellitus. Cochrane Database Syst Rev 2005 Apr 18;(2):CD003639.

20. Dormandy JA, Charbonnel B, Eckland DJ et al.; PROACTIVE Investigators. Secondary prevention of macrovascular events in patients with type 2 diabetes in the PROactive Study (PROspective pioglitAzone Clinical Trial In macroVascular Events): a randomised controlled trial. Lancet 2005;366(9493):1279-89.
21. Scirica BM, Bhatt DL, Braunwald E et al.; SAVOR-TIMI 53 Steering Committee and Investigators. Saxagliptin and cardiovascular outcomes in patients with type 2 diabetes mellitus. N Engl J Med 2013 Oct 3;369(14):1317-26.

22. White WB, Cannon CP, Heller SR et al.; EXAMINE Investigators. Alogliptin after acute coronary syndrome in patients with type 2 diabetes. N Engl J Med 2013 Oct 3;369(14):1327-35.

23. Green JB, Bethel MA, Armstrong PW et al.; TECOS Study Group. Effect of sitagliptin on cardiovascular outcomes in type 2 diabetes. N Engl J Med 2015 Jul 16;373(3):232-42.

24. Pfeffer MA, Claggett B, Diaz $\mathrm{R}$ et al.; ELIXA Investigators. Lixisenatide in patients with type 2 diabetes and acute coronary syndrome. N Engl J Med 2015 Dec 3;373(23):2247-57.

25. Marso SP, Daniels GH, Brown-Frandsen K et al.; LEADER Trial Investigators. Liraglutide and cardiovascular outcomes in type 2 diabetes. N Engl J Med 2016 Jul 28;375(4):311-22.

26. Marso SP, Bain SC, Consoli A et al.; SUSTAIN-6 Investigators. Semaglutide and cardiovascular outcomes in patients with type 2 diabetes. N Engl J Med 2016 Nov 10;375(19):1834-44.

27. Zinman B, Wanner C, Lachin JM et al.; EMPA-REG OUTCOME Investigators. Empagliflozin, cardiovascular outcomes, and mortality in type 2 diabetes. N Engl J Med 2015 Nov 26; 373(22):2117-28

28. Li L, Li S, Deng K et al. Dipeptidyl peptidase-4 inhibitors and risk of heart failure in type 2 diabetes: systematic review and metaanalysis of randomised and observational studies. BMJ 2016 Feb 17;352:1610.

29. Jardiance. Riassuntodelle Caratteristichedel Prodotto. Consultabilesu http://www.ema.europa.eu/docs/it_IT/document_library/EPAR_ Product_Information/human/002677/WC500168592.pdf

30. Neal B, Perkovic V, Mahaffey KW, et al; CANVAS Program Collaborative Group. Canagliflozin and cardiovascular and renal events in type 2 diabetes. N Engl J Med 2017 Jun 12. doi: 10.1056/ NEJMoa1611925.

31. Inzucchi SE, Zinman B, Wanner C et al. SGLT-2 inhibitors and cardiovascular risk: Proposed pathways and review of ongoing outcome trials. Diab Vasc Dis Res 2015 Mar;12(2):90-100.

32. Phung OJ, Schwartzman E, Allen RW et al. Sulphonylureas and risk of cardiovascular disease: systematic review and metaanalysis. Diabet Med 2013;30(10):1160-71.

33. Monami M, Genovese S, Mannucci E. Cardiovascular safety of sulfonylureas: a meta-analysis of randomized clinical trials Diabetes Obes Metab 2013;15(10):938-53.

34. Monami M, Dicembrini I, Mannucci E. Dipeptidyl peptidase-4 inhibitors and heart failure: a meta-analysis of randomized clinical trials. Nutr Metab Cardiovasc Dis 2014 Jul;24(7):689-97.

35. The Heart Outcomes Prevention Evaluation Study Investigators. Effects of an angiotensin-converting-enzyme inhibitor, ramipril on cardiovascular events in high-risk patients. $\mathrm{N}$ Engl J Med 2000;342:145-53.

36. Scandinavian Simvastatin Survival Study Group. Randomised 
trial of cholesterol lowering in 4444 patients with coronary heart disease: the Scandinavian Simvastatin Survival Study (4S). Lancet 1994;344(8934):1383-9.

37. Canadian Diabetes Association Clinical Practice Guidelines Expert Committee. Pharmacologic management of type 2 diabetes: 2016 Interim Update. Can J Diabetes 2016;40:484-6.

38. Ponikowski P, Voors AA, Anker SD et al.; Authors/Task Force Members. 2016 ESC Guidelines for the diagnosis and treatment of acute and chronic heart failure: The Task Force for the diagnosis and treatment of acute and chronic heart failure of the European Society of Cardiology (ESC) Developed with the special contribution of the Heart Failure Association (HFA) of the ESC. Eur Heart J 2016 Jul 14;37(27):2129-200, doi: 10,1093/eurheartj/ ehw128. Epub 2016 May 20.

39. Fitchett D, Zinman B, Wanner C et al.; EMPA-REG OUTCOME® trial Investigators. Heart failure outcomes with empagliflozin in patients with type 2 diabetes at high cardiovascular risk: results of the EMPA-REG OUTCOME® trial. Eur Heart J 2016 May 14;37(19):1526-34.

40. Stewart JH, McCredie MR, Williams SM et al; ESRD Incidence Study Group. Trends in incidence of treated end-stage renal disease, overall and by primary renal disease, in persons aged 20-64 years in Europe, Canada and the Asia-Pacific region, 19982002. Nephrology (Carlton) 2007;12:520-7.

41. Caramori L, Fioretto P, Mauer M. Enhancing the predictive value of urinary albumin for diabetic nephropathy. J Am Soc Nephrol 2006;17:339-52.

42. Magee GM, Bilous RW, Cardwell CR et al. Is hyperfiltration associated with the future risk of developing diabetic nephropathy? A meta-analysis. Diabetologia 2009;52(4):691-7.

43. Najafian B, Mauer M. Progression of diabetic nephropathy in type 1 diabetic patients. Diab Res Clin Pract 2009;83(1):1-8.

44. Satchell SC, Tooke JE. What is the mechanism of microalbuminuria in diabetes: a role for the glomerular endothelium? Diabetologia 2008;51(5):714-25.

45. Coca SG, Ismail-Beigi F, Haq $\mathrm{N}$ et al. Role of intensive glucose control in development of renal end points in type 2 diabetes mellitus: systematic review and meta-analysis intensive glucose control in type 2 diabetes. Arch Intern Med 2012;172:761-9.

46. Zoungas S, Chalmers J, Neal B et al.; ADVANCE-ON Collaborative
Group. Follow-up of blood-pressure lowering and glucose control in type 2 diabetes. N Engl J Med 2014;371:1392-406.

47. Tancredi M, Rosengren A, Svensson AM et al. Excess mortality among persons with type 2 diabetes. N Engl J Med 2015;373:1720-32.

48. Wanner C, Inzucchi SE, Lachin JM et al.; EMPA-REG OUTCOME Investigators. Empagliflozin and progression of kidney disease in type 2 diabetes. N Engl J Med 2016;375:323-34

49. Wanner C, et al. Annual Meeting of the American Society of Nephrology, 2015.

50. Riddle, Rosenstock J, Gerich J; Insulin Glargine 4002 Study Investigators. The treat-to-target trial: randomized addition of glargine or human NPH insulin to oral therapy of type 2 diabetic patients. Diabetes Care 2003;26(11):3080-6.

51. Rossetti P, Pampanelli S, Fanelli C et al. Intensive replacement of basal insulin in patients with type 1 diabetes given rapid-acting insulin analog at mealtime: a 3-month comparison between administration of NPH insulin four times daily and glargine insulin at dinner or bedtime. Diabetes Care 2003;26(5):1490-6.

52. Yki-Järvinen, Kauppinen-Mäkelin R, Tiikkainen $M$ et al. Insulin glargine or NPH combined with metformin in type 2 diabetes: the LANMET study. Diabetologia 2006;49(3):442-51.

53. ORIGIN Trial Investigators; Bosch J, Gerstein HC, Dagenais GR et al. Basal insulin and cardiovascular and other outcomes in dysglycemia. N Engl J Med 2012;367(4):319-28.

54. Blevins TC, Dahl D, Rosenstock J et al. Efficacy and safety of LY2963016 insulin glargine compared with insulin glargine (Lantus $®$ ) in patients with type 1 diabetes in a randomized controlled trial: the ELEMENT 1 study. Diabetes Obes Metab 2015;17:726-33.

55. Rosenstock J, Hollander P, Bhargava A et al. Similar efficacy and safety of LY2963016 insulin glargine and insulin glargine (Lantus $®$ ) in patients with type 2 diabetes who were insulinnaïve or previously treated with insulin glargine: a randomized, double-blind controlled trial (the ELEMENT 2 study). Diabetes Obes Metab 2015 Aug;17(8):734-41.

56. Panos Kanavos, Stacey van den Aardweg and Willemien Schurer Diabetes expenditure, burden of disease and management in 5 EU countries. LSE Health, London School of Economics. Consultabile su: http://www.lse.ac.uk/LSEHealth AndSocialCare/research/LSEHealth/MTRG/LSEDiabetes Report26Jan2012.pdf 